\title{
Long noncoding RNA PVT1 promotes tumor cell proliferation, invasion, migration and inhibits apoptosis in oral squamous cell carcinoma by regulating miR-150-5p/GLUT-1
}

\author{
$\mathrm{XIALI}^{1}$ and HENGJIE REN ${ }^{2}$ \\ Departments of ${ }^{1}$ Stomatology and ${ }^{2}$ Nursing, The First Affiliated Hospital of Jinzhou Medical University, \\ Jinzhou, Liaoning 121001, P.R. China
}

Received October 21, 2019; Accepted May 27, 2020

DOI: $10.3892 / o r .2020 .7706$

\begin{abstract}
Oral squamous cell carcinoma (OSCC) is a cancer with high morbidity and mortality. Research has demonstrated that long non-coding RNAs (IncRNAs) are critical for tumor initiation and development. In the present study, we aimed to ascertain the functions and potential mechanisms of lncRNA plasmacytoma variant translocation 1 (PVT1) in OSCC. Firstly, we found that the expression of PVT1 was increased in human OSCC tumor tissues and it was related to reduced survival of the patients. Furthermore, miR-150-5p expression was downregulated in OSCC tumor tissues and it was negatively related with PVT1. Moreover, GLUT-1 protein expression was upregulated in human OSCC tumor tissues. In addition, cell proliferation capacity was measured by CCK- 8 assay and cell invasion and migration were measured by Transwell assay. PVT1 overexpression promoted cell proliferation, invasion and migration, while these effects were abrogated by PVT1 downregulation. In addition, luciferase gene reporter assay verified the miR-150-5p directly binds with PVT1, which regulates the biological functions of OSCC. Additionally, luciferase gene reporter assay confirmed that GLUT-1 was a target for miR-150-5p. The promotion of cell proliferation, invasion and migration in LV-PVT1-transfected cells was eliminated following miR-150-5p overexpression. Finally, in vivo nude mouse xenograft model further verified that PVT1 knockdown inhibited tumor growth, formation, invasion and migration. According to the results, PVT1 is increased in human OSCC tumor tissues, and is related to the poor prognosis of human OSCC patients. We uncovered a previously unappreciated PVT1/miR-150-5p/GLUT-1 signaling axis that promotes cell proliferation, invasion, migration and inhibits
\end{abstract}

Correspondence to: Dr Hengjie Ren, Department of Nursing, The First Affiliated Hospital of Jinzhou Medical University, 2 Renmin Street, Guta, Jinzhou, Liaoning 121001, P.R. China

E-mail: renhengjie88@126.com

Key words: 1ncRNA PVT1, miR-150-5p, GLUT-1, oral squamous cell carcinoma, OSCC apoptosis in OSCC cell lines and in vivo, which suggests that this axis could be a target for the treatment of OSCC.

\section{Introduction}

Oral squamous cell carcinoma (OSCC) is a malignant epithelial cancer that threatens the health of individuals worldwide and OSCC frequently occurs in the tongue and buccal mucosa (1-3). The 5-year survival for human OSCC patients is approximately $50 \%$ and the incidence of OSCC is still increasing with a reported annual incidence worldwide of approximately 275,000 (4,5) and 50,000 in China (6). However, the detailed mechanisms of the tumor formation, progression and metastasis of OSCC require further in-depth research. Therefore, it has been a difficult quest for researchers to elucidate the mechanisms of OSCC progression and metastasis and to identify new methods for the early diagnosis and treatment for OSCC.

Long non-coding RNAs (lncRNAs) are a diverse class of RNA transcripts longer than 200 nucleotides that have limited protein coding capacity $(7,8)$. IncRNAs have been found to participate in various biological processes in cancers such as cervical cancer, prostate carcinoma, epithelial ovarian cancer and breast cancer, including tumorigenesis, metastasis and chemotherapy resistance (9-12). Recent evidence shows that IncRNAs play oncogenic or tumor-suppressor roles in OSCC (13-15). For example, LINC01133 was found to inhibit OSCC metastasis by regulating GDF15 (14). Zhu et al demonstrated that the upregulation of 1ncHAS2-AS1 plays important roles in the invasiveness of OSCC (13). Jin et al found that IncRNA MORT inhibited cell proliferation by repressing ROCK1 in OSCC (15). IncRNA plasmacytoma variant translocation 1 (PVT1) has been demonstrated to be increased and to function as an oncogenic gene to promote tumor formation, invasion, migration and resistance in many types of cancers (16-21), such as glioma (16), colorectal cancer (17), gastric cancer (18), ovarian cancer (19) and gallbladder cancer (20). However, the roles of PVT1 in OSCC remain unknown.

MicroRNAs (miRNAs/miRs) are approximately 18-22 nucleotides in length and cannot be translated into protein as well, yet miRNAs are verified to play critical roles 
in the biological functions of various diseases via directly targeting downstream target genes (22-26). Salmena et al firstly reported that lncRNAs interact with miRNAs through 'competing endogenous RNAs' (ceRNAs), which indicate that lncRNAs interact with miRNAs $(27,28)$ and this hypothesis has been proven in various pathological conditions and diseases (29-33). miR-150-5p was found to participate in the biological processes of various cancers (34-36). For example, miR-150-5p is important in inhibiting the metastasis of human non-small cell lung cancer (NSCLC) (35). Chen et al demonstrated that miR-150-5p repressed progression of colorectal cancer (36). Lu et al revealed that miR-150-5p participated in the processes of proliferation, migration and invasion in NSCLC (34). However, whether it plays similar roles in OSCC remains unknown.

In the present study, we investigated expression levels of PVT1 in OSCC tissues and adjacent tissues. Then we observed cell proliferation, invasion and migration in OSCC cells after PVT1 was overexpressed and downregulated. Bioinformatics predicted that PVT1 may competitively bind with miR-150-5p, which could then target by binding with glucose transporter type 1 (GLUT1). GLUT1 was reported to be an oncogenic gene that was associated with tumor cell proliferation, tumorigenesis, glucose metabolism and resistance in some cancers, such as urinary bladder, non-small cell lung and prostate cancer (37-39). We uncovered that expression of PVT1 was increased in human OSCC patients and cell lines. As a result, we aimed to ascertain the functions of PVT1 in OSCC. The results revealed that PVT1 expression is increased in OSCC tissues and it plays some roles in OSCC, such as regulating cell proliferation, cell apoptosis, invasion and migration in OSCC.

\section{Materials and methods}

Patient tissues. A total of 70 paired cancer and adjacent tissues were collected from patients with OSCC from October 2012 to September 2013 at the First Affiliated Hospital of Jinzhou Medical University (Jinzhou, Liaoning, China). Totally, 34 male and 36 female patients were recruited and their mean age was $48.4 \pm 10.3$ years. All tissues were cut into small pieces approximately $40 \mathrm{mg}$ and frozen at $-80^{\circ} \mathrm{C}$. Two groups were divided according to the mean expression of PVT1: PVT1 high expression and low expression groups. The Declaration of Helsinki was consulted during the human study. We received informed consent from all patients and the study was approved by the Faculty of Medicine's Ethics Committee of The First Affiliated Hospital of Jinzhou Medical University (ethical approval no. JYD160923).

Cell culture. Human normal oral epithelial cell line NOK and OSCC cell lines, including SCC-090, SCC-25 and CAL-27 were purchased from the American Type Culture Collection (ATCC). The cells were cultured in Dulbecco's modified Eagle's medium (DMEM; Invitrogen; Thermo Fisher Scientific, Inc.) with $10 \%$ fetal bovine serum (FBS; Gibco; Thermo Fisher Scientific, Inc.), penicillin (100 U/ml) and streptomycin $(100 \mu \mathrm{g} / \mathrm{ml})$. The cells were cultured in an incubator at $37^{\circ} \mathrm{C}$ with $5 \% \mathrm{CO}_{2}$.

Construction of lentivirus and cell transfection. The full length of human PVT1 cDNA was constructed into a lentivirus
(Shanghai GenePharma Co., Ltd.), resulting in PVT1 overexpression, and was named LV-PVT1 and the negative control (LV-NC) was obtained from GenePharma. Furthermore, PVT1-shRNA (LV-sh PVT1) was synthesized and cloned into a lentivirus, resulted in PVT1 downregulation and the shRNA negative control (LV-sh NC) was obtained from GenePharma. The sequences were as follow: PVT1-shRNA, 5'-CCUGAUGGAUUUACAGUGATT-3' and NC-shRNA, 5'-GCUACGAUCUGCCUAAGAUTT-3'. LV-PVT1 or LV-NC or LV-sh PVT1 or LV-sh NC was respectively infected into SCC-25 and CAL-27 cells according to the manufacturer's instructions. After antibiotic selection and constructed for 2 weeks, the stable cells with PVT1 overexpression or downregulation were obtained. Cells were plated in 6-well plates (1x10\%/well) until reaching 60-70\% confluence. Transfection reagent Lipofectamine 2000 (Invitrogen; Thermo Fisher Scientific, Inc.), serum-free DMEM and hsa-miR-150-5p mimics (Guangzhou RiboBio Co., Ltd, miR10000451-1-5, $5 \mathrm{nmol}$ ) were mixed and incubated at room temperature for $30 \mathrm{~min}$, which were added into the prepared 6-well plates with complete medium with $10 \%$ FBS.

$C C K-8$ assay. Cell proliferation abilities were examined using CCK-8 assay (Dojundo) in accordance with the supplier's protocol. Cells $\left(1 \times 10^{3} /\right.$ well) were plated in 96 -well plates and transfected with the indicated lentivirus and mimics for 24 h. Each group was set with three replicate wells. For each well, $10 \mu \mathrm{l}$ CCK-8 was added at $0,1,2$ and 3 days, and then incubation was carried out in darkness for another $3 \mathrm{~h}$ at room temperature. Finally, the absorbance (OD) value was measured at $450 \mathrm{~nm}$ with a microplate reader.

Cell invasion and migration assays. Cells $\left(2 \times 10^{5}\right.$ cells/well $)$ were diluted in $200 \mu \mathrm{l}$ serum-free DMEM and inoculated onto the upper wells of a Transwell chamber coated with $20 \mu$ l Matrigel (BD Biosciences). DMEM with 10\% FBS was added into the lower chambers, which were incubated for $48 \mathrm{~h}$. Then the non-invasive cells were removed by using a cotton swab following overnight incubation. The invasive cells in the lower chambers were fixed and stained with $100 \%$ pre-cold methanol and $0.1 \%$ crystal violet at room temperature for $30 \mathrm{~min}$. Finally, the cells were observed by using an inverted light microscope (magnification, x200, Olympus Corp.). For the cell migration assay, cells were suspended in FBS-free DMEM with $1 \mu \mathrm{g} / \mathrm{ml}$ Mitomycin C and plated in the upper wells of the Transwell chamber and DMEM with 10\% FBS was mixed into the lower chambers. Then the following steps were similar to the invasion assay. Five visual fields of each chamber were randomly chosen and cell number was counted under a light microscope (magnification x100; Olympus Corp.; Japan Nikon Corp.).

$R N A$ extraction and reverse transcription-quantitative $P C R$ $(R T-q P C R)$. Total RNA of tissues and cells were extracted by using Trizol (Invitrogen; Thermo Fisher Scientific, Inc.) according to the manufacturer's protocol. For the quantitative detection of genes, RT was conducted at $37^{\circ} \mathrm{C}$ for $15 \mathrm{~min}$, which was followed by $85^{\circ} \mathrm{C}$ for $5 \mathrm{sec}$ with a PrimeScript ${ }^{\mathrm{TM}} \mathrm{RT}$ Reagent Kit (Takara Bio, Inc.) according to the manufacturer's protocol. PCR primers were purchased from ShangHai Gene 
Pharma and mRNA expression was detected by SYBR Premix Ex Taq II (Takara Bio, Inc.). Two-step PCR amplification was performed at $95^{\circ} \mathrm{C}$ for $180 \mathrm{sec}$, which were then followed by 40 cycles at $95^{\circ} \mathrm{C}$ for $10 \mathrm{sec}$ and $60^{\circ} \mathrm{C}$ for $30 \mathrm{sec}$. Final extension was performed at $72^{\circ} \mathrm{C}$ for $5 \mathrm{~min}$. Expression levels were normalized to $\beta$-actin and U6. The $2^{-\Delta \Delta C q}$ method (40) was used to calculate the relative gene expression. Gene primers are listed in Table I.

Protein extraction and western blot analysis. Total proteins of tissues, mouse tumors and cells were extracted by using $150 \mu 1$ PRO-PREP Protein Extraction Solution (iNtRON), which were measured by BCA protein assay kit (Beyotime Institute of Biotechnology). Protein $(40 \mu \mathrm{g})$ was added to $10 \%$ SDS-PAGE and the separated protein was transferred onto polyvinylidene fluoride membranes (PVDF; EMD Millipore), which were blocked by $5 \%$ non-fat milk for $1 \mathrm{~h}$. Then TBST (Boster) was used to wash the membranes for three times, which were incubated with primary antibodies overnight at $4^{\circ} \mathrm{C}$, which were purchased from Abcam. The antibodies used in the western blot analysis are listed in Table II. After that, they were incubated with matched secondary antibodies (goat anti-rabbit, dilution 1:5,000, product code ab150077; goat anti-mouse, dilution 1:5,000; product code ab150113) for $1 \mathrm{~h}$. Finally, the proteins were visualized and quantified by using Pierce ECL Western blot substrate (Thermo Fisher Scientific, Inc.) with ECL detection system (Thermo Fisher Scientific, Inc.) and proteins were normalized to $\beta$-actin. The gray value of the target band was analyzed by using Image Lab (version 3.1; Bio-Rad Laboratories, Inc.).

Luciferase assay. StarBase v2.0 database (41) was used to analyze the potential miRNAs that interact with PVT1. miR-150-5p was identified as a potential miRNA. Human PVT1-3'-untranslated region (3'-UTR) and GLUT-1-3'-UTR reporter constructs containing the potential binding site of miR-150-5p, and their identical sequences with a mutation in the seed sequence, which were constructed into pmiR-GLO-basic reporter vector (Promega Corp.). Cells $\left(2 \times 10^{5}\right.$ cells/well) were plated into 48 -well plates for $12 \mathrm{~h}$, co-transfected with the indicated plasmids and miR-150-5p mimics for $24 \mathrm{~h}$. The plasmids (200 ng) were mixed with Lipofectamine 2000 (Invitrogen; Thermo Fisher Scientific, Inc.), Renilla luciferase plasmids and DMEM for $30 \mathrm{~min}$ and then added into the prepared cells for $24 \mathrm{~h}$. Finally, the luciferase activities were measured by the Dual-Luciferase Assay kit (Promega Corp.) according to the manufacturer's instructions. Every sample was tested for three times and the whole experiment was repeated for three times.

In vivo nude mouse xenograft assay. Twelve female $\mathrm{BALB} / \mathrm{c}$ nude mice, about 4-weeks of age and weighing 15-20 g, were purchased from the Animal Center of Shanghai Jiaotong University (Shanghai, China), and were housed in humidityand temperature-controlled rooms $\left(40-80 \% ; 22 \pm 2^{\circ} \mathrm{C}\right)$ for 7 days prior to the experiments. All animal handling and experimental procedures were approved by the Animal Ethics Committees of the First Affiliated Hospital of Jinzhou Medical University and in accordance with the guidelines of the China Council of Animal Care. Two groups were randomly divided,
Table I. Sequences of primers for qPCR.

\begin{tabular}{ll}
\hline Genes & \multicolumn{1}{c}{ Primer sequences } \\
\hline$P V T 1$ & F: 5'-TGAGAACTGTCCTTACGTGACC-3' \\
& R: 5'-AGAGCACCAAGACTGGCTCT-3' \\
$m i R-150-5 p$ & F: 5'-CGGCGGCAAAGTGCTTACAG-3' \\
& R: 5'-GGGCATACATCGGCTAATACA-3' \\
GLUT-1 & F: 5'-GTCAACACGGCCTTCACTG-3' \\
& R: 5'-GGTCATGAGTATGGCACAACC-3' \\
$\beta$-actin & F: 5'-CCAAGGCCAACCGCGAGAAGAT-3' \\
& R: 5'-AGGGTACATGGTGGTGCCGCCA-3' \\
$U 6$ & F: 5'-CGCTTCGGCAGCACATATACT-3' \\
& R: 5'-CGCTTCACGAATTTGCGTGTC-3'
\end{tabular}

F, forward; R, reverse; PVT1, lncRNA plasmacytoma variant translocation 1; GLUT1, glucose transporter 1; qPCR, real-time quantitative PCR.

as the LV-NC and LV-shPVT1 group. Mice in each group were injected subcutaneously on the right axilla with $1 \times 10^{7} / 0.1 \mathrm{ml}$ of SCC-25 cells transfected with LV-shPVT1 or LV-NC. After successful transplantation, the tumor volumes of nude mice in two groups were observed every week. The tumor volume (V) was calculated as $\mathrm{V}=\pi / 6 \mathrm{x}$ length $\mathrm{x}$ width $\mathrm{x}$ height. After 35 days, mice were sacrificed with isoflurane (4\%) for $1 \mathrm{~min}$ and cervical dislocation was performed to verify euthanasia, finally, the tumor tissues were obtained.

Statistical analysis. All data were analyzed by SPSS 19.0 (IBM Corp.) and GraphPad Prism 6.0 (GraphPad Software). Student's t-test was used to analyze the statistical significance between two groups, and one-way ANOVA and Tukey's post hoc test method were used to analyze the statistical significance among more than two groups, while Dunnett's test was used when each group was only compared to the control group. Count data were processed by Chi-square test. Correlations between PVT1, miR-150-5p and GLUT-1 were analyzed by using Pearson's correlation analysis. Survival curves were analyzed by using Kaplan-Meier survival test. $\mathrm{P}<0.05$ was considered to indicate a statistically significant difference.

\section{Results}

PVT1 is upregulated in OSCC tumor tissues and cell lines. First, we determined the expression of PVT1 in 70 cases of OSCC tissues and adjacent tissues. PVT1 was increased 2.2-fold in OSCC tissues when compared to the adjacent tissues (Fig. 1A) $(\mathrm{P}<0.001)$. Furthermore, we collected and analyzed the correlations between PVT1 and clinicopathological features in the OSCC patients. PVT1 upregulation was correlated with advanced TNM stage, metastasis and recurrence (Table III) $(\mathrm{P}<0.05)$. Furthermore, we found that PVT1 was increased 1.7-fold in the OSCC tissues with metastasis $(n=19)$, compared to that in OSCC tissues without metastasis $(\mathrm{n}=51)($ Fig. 1B) $(\mathrm{P}<0.001)$. We also divided patients into two groups according to the TNM stage, and the results showed that the expression 
Table II. Antibodies used in western blot analysis.

\begin{tabular}{|c|c|c|c|c|}
\hline Proteins & Host & $\mathrm{kDa}$ & Catalog no. & Antibody dilution \\
\hline Ki67 & Rabbit & 359 & ab16667 & $1: 5,000$ \\
\hline Bcl-2 & Rabbit & 26 & ab182858 & $1: 1,000$ \\
\hline Bax & Rabbit & 21 & ab32503 & $1: 1,000$ \\
\hline Cleaved caspase- 3 & Rabbit & 17 & ab49822 & $1: 500$ \\
\hline MMP-9 & Rabbit & 92 & ab38898 & $1: 1,000$ \\
\hline MMP-14 & Rabbit & 66 & ab51074 & $1: 2,000$ \\
\hline GLUT-1 & Rabbit & 55 & ab40084 & $1: 5,000$ \\
\hline$\beta$-actin & Rabbit & 42 & ab179467 & $1: 5,000$ \\
\hline
\end{tabular}

Bcl-2, B-cell lymphoma 2; Bax, BCL2-associated X protein; MMP, matrix metalloproteinase; GLUT1, glucose transporter type 1.

Table III. Association between PVT1 expression and the clinicopathological features of the OSCC patients.

\begin{tabular}{|c|c|c|c|}
\hline Parameters & Low expression $(n=35)$ & High expression $(n=35)$ & P-value \\
\hline Sex & & & 0.339 \\
\hline Male & 15 & 19 & \\
\hline Female & 20 & 16 & \\
\hline Age (years) & & & 0.147 \\
\hline$<45$ & 18 & 12 & \\
\hline$\geq 45$ & 17 & 23 & \\
\hline TNM stage & & & 0.016 \\
\hline $\mathrm{I}$ & 15 & 5 & \\
\hline II & 10 & 8 & \\
\hline III & 7 & 12 & \\
\hline IV & 3 & 10 & \\
\hline Metastasis & & & 0.015 \\
\hline Yes & 5 & 14 & \\
\hline No & 30 & 21 & \\
\hline Recurrence & & & 0.006 \\
\hline Yes & 7 & 18 & \\
\hline No & 28 & 17 & \\
\hline
\end{tabular}

OSCC, oral squamous cell carcinoma; PVT1, lncRNA plasmacytoma variant translocation 1. P-values in bold print indicate significantly significant differences.

levels of PVT1 in stage III-IV were significantly higher than those in stage I-II (Fig. 1C) $(\mathrm{P}<0.001)$. To explore the prognostic values of PVT1 in OSCC patients, Kaplan-Meier analysis was performed. Results revealed that patients with high expression of PVT1 had a worse recurrence-free and overall survival when compared with patients in the low PVT1 expression group (Fig. 1D and E). Then we detected PVT1 expression in human normal oral epithelial cell line NOK and OSCC cell lines, including SCC-090, SCC-25 and CAL-27. The results revealed that expression of PVT1 was significantly increased in the human OSCC cells when compared to that in the NOK cells (Fig. 1F). As the expression levels of PVT1 in SCC-25 and CAL-27 were much higher than other cell lines, therefore, we chose
SCC-25 and CAL-27 cell lines for subsequent experiments. These data indicated that PVT1 was upregulated in OSCC, and was correlated with a poor prognosis of OSCC patients; however, the detailed mechanisms remained unknown.

Overexpression of $P V T 1$ promotes cell proliferation, invasion, migration and inhibits apoptosis in OSCC cells. To explore the functions of PVT1 in OSCC, LV-PVT1 was constructed, which resulted in PVT1 overexpression. After LV-PVT1 infection, PVT1 expression was significantly increased compared to the LV-NC group (Fig. 2A) ( $<<0.001)$. CCK-8 assay showed that PVT1 upregulation significantly promoted the cell proliferation of SCC-25 and CAL-27 cells at 3 and 5 days (Fig. 2B and C). Furthermore, Transwell invasion assays 

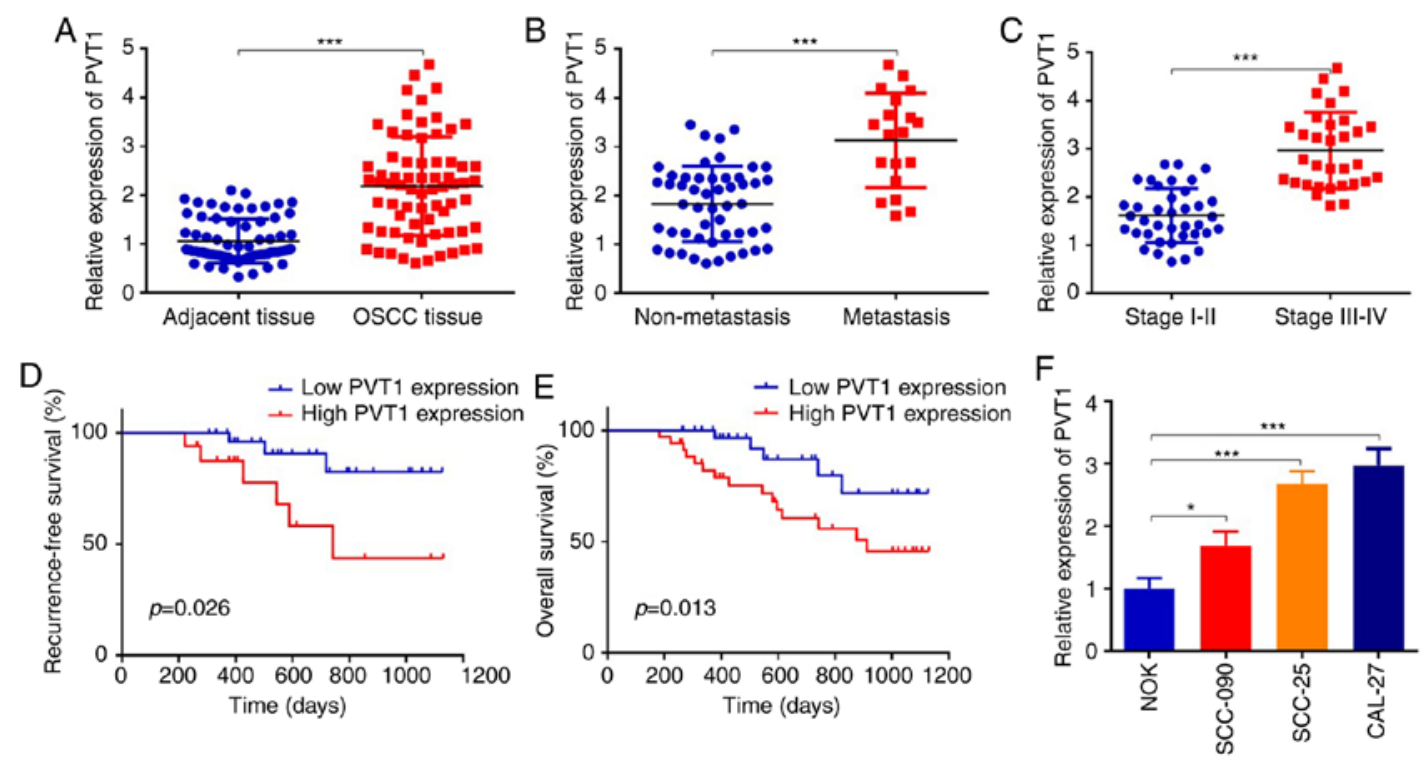

Figure 1. PVT1 is upregulated in OSCC tissues and cell lines. (A) qPCR indicated that PVT1 was upregulated in OSCC tissues (n=70) compared to adjacent tissues $(\mathrm{n}=70)$. (B) qPCR revealed that PVT1 was upregulated in metastasis patients $(\mathrm{n}=19)$ when compared with patients without metastasis $(\mathrm{n}=51)$. $(\mathrm{C}) \mathrm{qPCR}$ revealed that PVT1 in patients at stage III-IV was higher than at stage I-II. (D and E) Kaplan-Meier survival analysis demonstrated that recurrence-free and overall survival rates were much lower in patients with PTV1 high expression $(n=35)$ compared with those with PTV1 low expression $(n=35)$. (F) qPCR showed that PVT1 was increased in OSCC cell lines when compared to human normal oral epithelial NOK cells ( ${ }^{*} \mathrm{P}<0.05$ and $\left.{ }^{* * * *} \mathrm{P}<0.001\right)$. OSCC, oral squamous cell carcinoma; PVT1, IncRNA plasmacytoma variant translocation 1; qPCR, real-time quantitative PCR.

indicated that increased expression of PVT1 significantly enhanced SCC-25 and CAL-27 cell invasion (Fig. 2D and E) $(\mathrm{P}<0.001)$, and the same results were found for the migration assays (Fig. 2F and G) $(\mathrm{P}<0.001)$. Moreover, we detected apoptotic-associated protein expression, such as Ki67, Bcl-2, Bax and cleaved caspase 3, and also detected expression levels of migration-associated proteins, such as MMP-9 and MMP-14 in the OSCC cell lines SCC-25 and CAL-27. Western blot analysis demonstrated that protein expression of Ki67 and Bcl-2 were significantly increased, the apoptotic genes Bax and cleaved caspase 3 were significantly decreased and migration-related MMP-9 and MMP-14 were significantly increased after LV-PVT1 infection (Fig. 2H-K). Collectively, these data indicated that overexpression of PVT1 enhanced cell proliferation, invasion, migration and inhibited apoptosis in OSCC cells.

Downregulation of PVT1 suppresses cell proliferation, invasion, migration and promotes apoptosis in OSCC. To further verify the functions of PVT1 in OSCC SCC-25 and CAL-27 cell lines, the LV-shPVT1 was constructed, which resulted in PVT1 downregulation. After LV-sh PVT1 infection into SCC-25 and CAL-27, the PVT1 levels were significantly inhibited compared with the cells transfected with LV-sh NC (Fig. 3A) $(\mathrm{P}<0.001)$. CCK-8 assay revealed that PVT1 downregulation significantly suppressed cell proliferation compared with the LV-sh NC (Fig. 3B and C). Furthermore, Transwell assays indicated that downregulation of PVT1 significantly inhibited cell invasion and migration (Fig. 3D-G) $(\mathrm{P}<0.001)$. Moreover, protein expression levels of Ki67 and Bcl-2 were significantly decreased, the apoptotic genes Bax and cleaved caspase 3 were upregulated and the migration-associated genes MMP-9 and MMP-14 were significantly inhibited following with LV-shPVT1 infection when compared with the LV-sh NC group (Fig. 3H-K). Collectively, these data indicate that PVT1 is vitally important in cell apoptosis, proliferation, invasion and migration in OSCC. However, the detailed mechanisms of PVT1 in tumorigenesis, invasion and migration in OSCC remain unknown.

PVT1 directly sponges miR-150-5p in OSCC. To further explore the detailed mechanism involved in the participation of PVT1 in the processes of cell proliferation, invasion and migration in OSCC, starBase v2.0 database (41) was used and miR-150-5p was found to be a potential downstream miRNA that contains potential binding sites with PVT1. Then the expression of miR-150-5p in patients was detected. The results revealed that miR-150-5p was significantly suppressed in OSCC tumor tissues when compared with that in the adjacent tissues (Fig. 4A) $(\mathrm{P}<0.001)$. Furthermore, miR-150-5p was decreased 2.0 -fold in OSCC patients with metastasis $(n=19)$, compared to those without metastasis $(n=51)$ (Fig. 4B) $(\mathrm{P}<0.001)$. Moreover, expression of miR-150-5p in stage III-IV tissues was much lower than expression in stage I-II tissues (Fig. 4C) $(\mathrm{P}<0.001)$. In addition, correlation analysis was performed between PVT1 and miR-150-5p, which showed that miR-150-5p was negatively correlated with PVT1 in OSCC and metastatic patients (Fig. 4D and E). We also detected miR-150-5p expressions in NOK and OSCC cells. The results revealed that miR-150-5p was significantly suppressed in the OSCC cell lines when compared with NOK cells (Fig. 4F). In addition, expression of miR-150-5p was significantly suppressed in SCC-25 and CAL-27 cells following LV-PVT1 infection $(\mathrm{P}<0.01)$, while they were significantly upregulated following LV-shPVT1 infection $(\mathrm{P}<0.001)$, when compared with the relevant $\mathrm{NC}$ group. (Fig. $4 \mathrm{G}$ and $\mathrm{H}$ ). Above all, these results indicate that PVT1 is negatively interactive with miR-150-5p, which may be a downstream factor of PVT1 

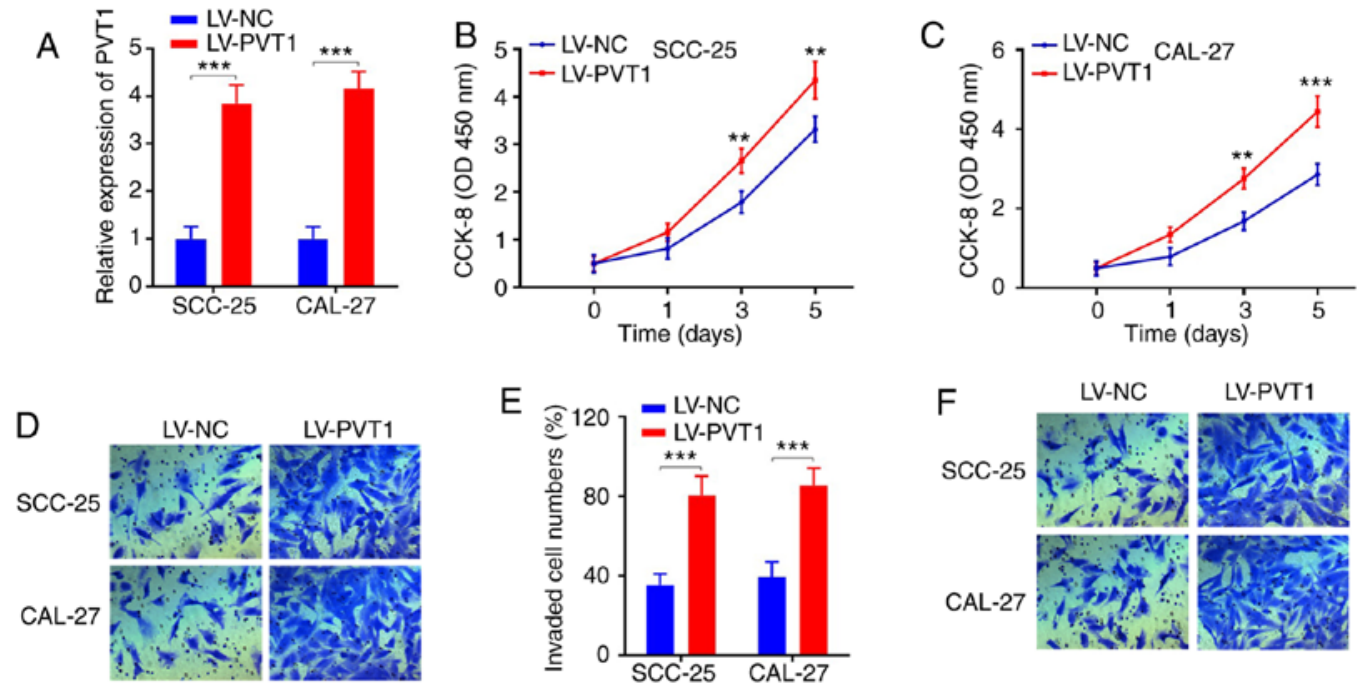

$\mathrm{E}$
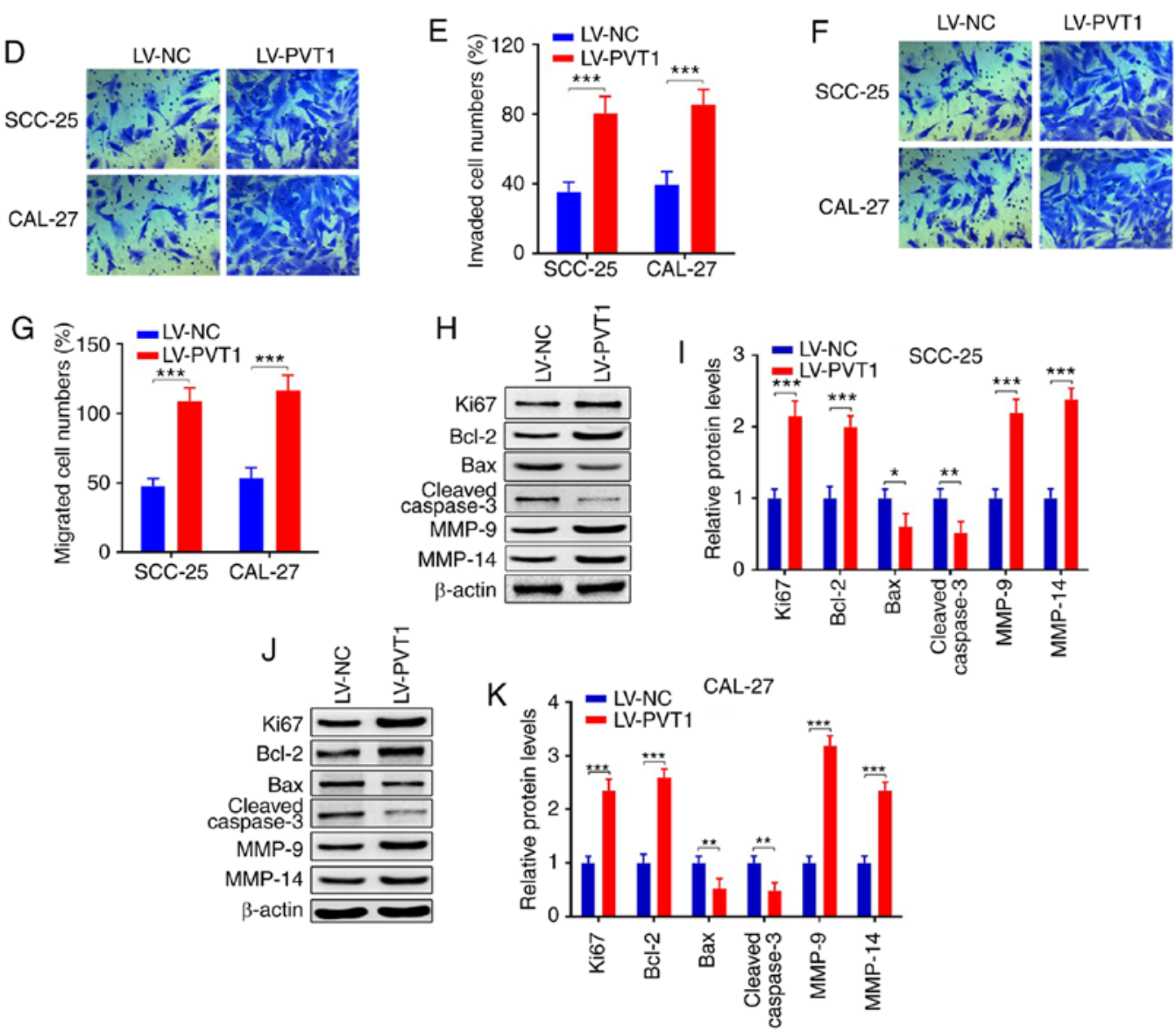

Figure 2. Overexpression of PVT1 promotes cell proliferation, invasion, migration and inhibits apoptosis in OSCC. (A) qPCR showed that PVT1 expression was upregulated in SCC-25 and CAL-27 cells transfected with LV-PVT1 when compared with the LV-NC group. (B and C) CCK-8 assay revealed that the proliferation abilities were promoted in cell lines transfected with LV-PVT1 when compared with the LV-NC group. (D-G) Transwell assays indicated that invasion and migration were both enhanced in cell lines transfected with LV-PVT1 when compared with the LV-NC group. (H-K) Protein expression levels of Ki67, Bcl-2, Bax, cleaved caspase 3, MMP-9 and MMP-14 were detected by western blot analysis in SCC-25 and CAL-27 cell lines transfected with LV-PVT1 when compared with the LV-NC group $\left({ }^{*} \mathrm{P}<0.05 ;{ }^{* *} \mathrm{P}<0.01,{ }^{* * *} \mathrm{P}<0.001\right)$. OSCC, PVT1, IncRNA plasmacytoma variant translocation 1; OSCC, oral squamous cell carcinoma; Bcl-2, B-cell lymphoma 2; Bax, bcl-2-like protein 4; MMP, matrix metalloproteinase; qPCR, real-time quantitative PCR.

(Fig. 4I). To confirm whether PVT1 could competitively bind with miR-150-5p, wild-type WT-PVT1 and mutant MUT-PVT1 sequences were constructed into vectors and the luciferase gene reporter assay was performed. Results showed that relative luciferase activity in SCC- 25 cells co-transfected with WT-PVT1 and miR-150-5p mimics was obviously repressed. However, it was reversed following co-transfected with MUT-PVT1 and miR-150-5p mimics. And the same result was found in CAL-27 cells (Fig. 4J and K). These data indicate that PVT1 competitively binds with miR-150-5p in OSCC.

miR-150-5p inhibits cell proliferation, invasion, migration and promotes apoptosis in OSCC. We aimed to further ascertain the roles of miR-150-5p in OSCC. miR-150-5p mimics was respectively transfected into SCC- 25 and CAL- 27 cells. The results revealed that miR-150-5p was significantly increased following transfection with miR-mimics when compared with the miR-mimics NC group (Fig. 5A) $(\mathrm{P}<0.001)$. In addition, the cell proliferation abilities were significantly suppressed in the SCC-25 and CAL-27 cell lines following transfection with miR-150-5p mimics compared with the miR-mimics NC group (Fig. 5B and C) $(\mathrm{P}<0.01)$. Transwell assays revealed that cell invasion and migration abilities were significantly suppressed following miR-150-5p overexpression (Fig. 5D-G) $(\mathrm{P}<0.001)$. Protein expression levels of Ki67 and Bcl-2 were significantly inhibited, apoptotic genes Bax and cleaved caspase 3 were upregulated, and migration and invasion-associated genes 
A
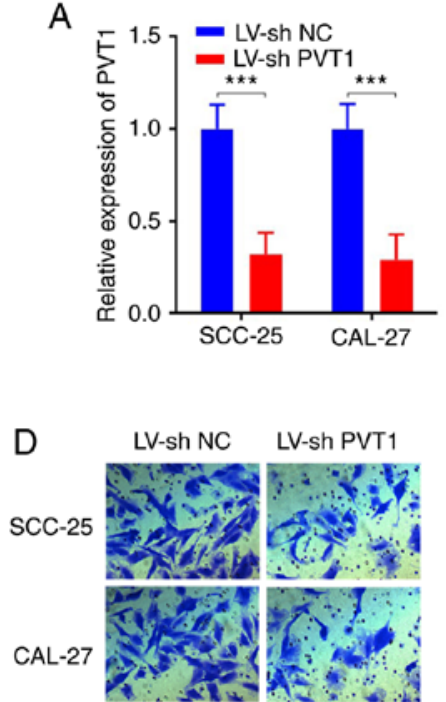

B
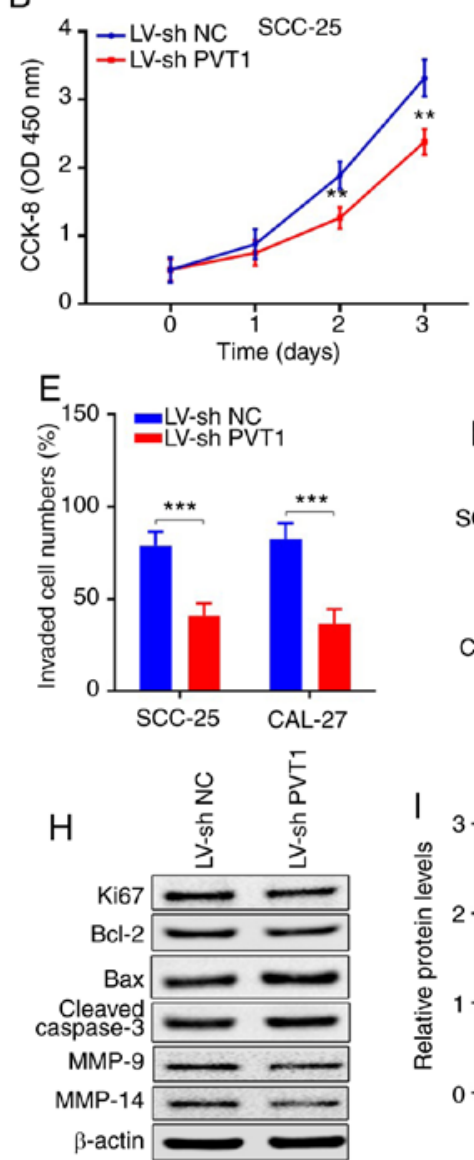

$\beta$-actin
C
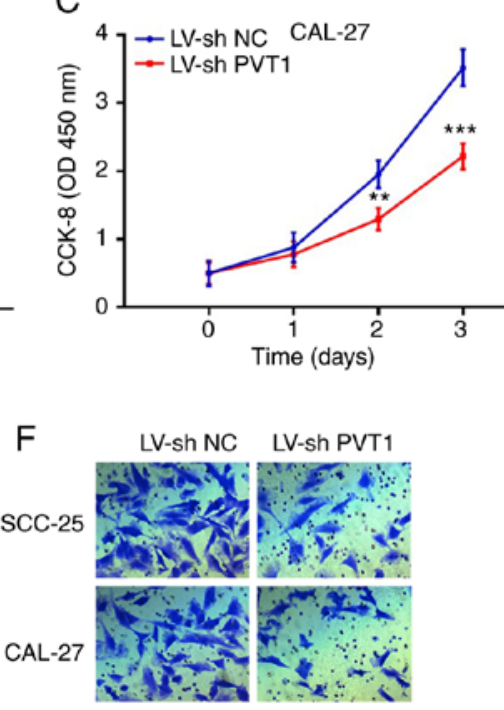
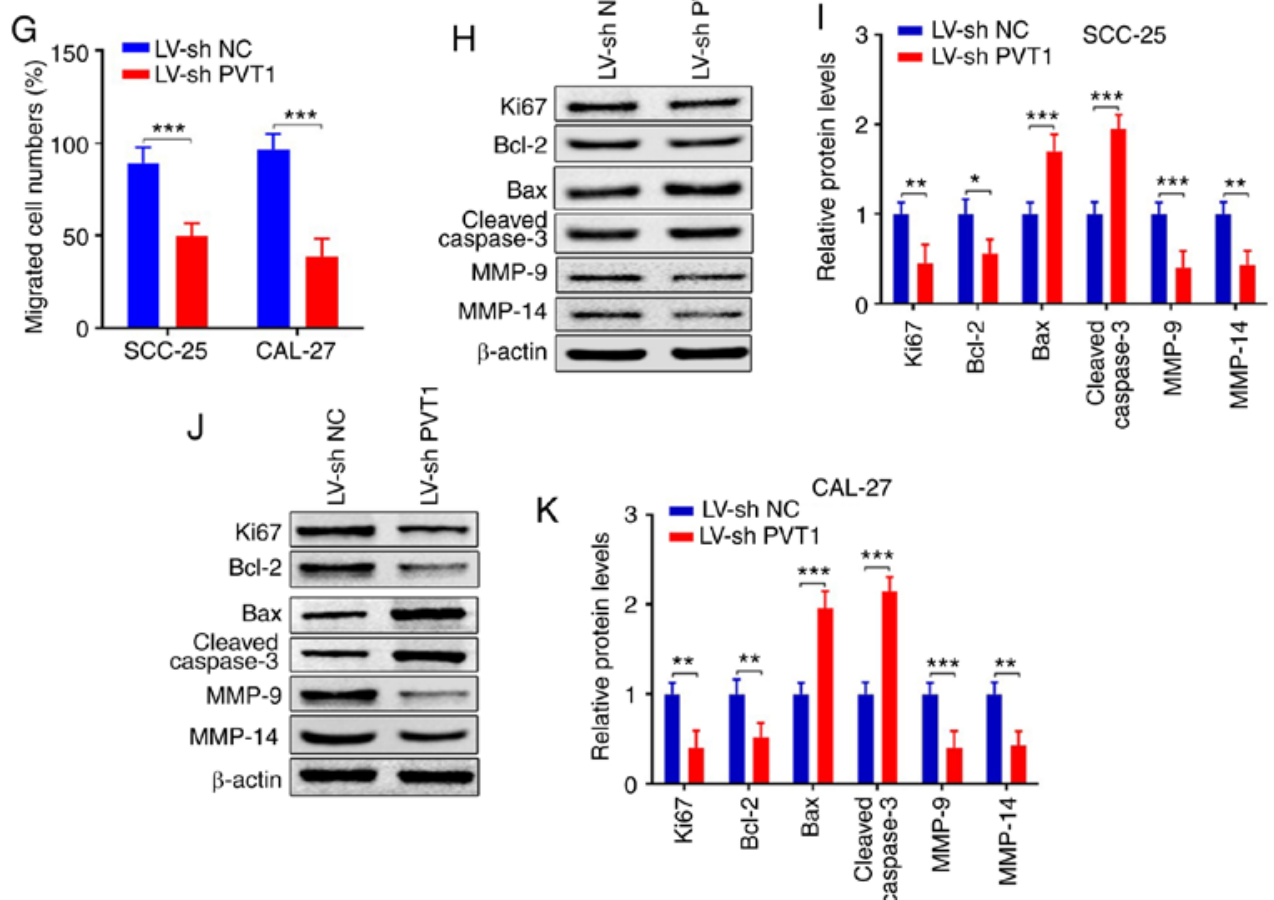

Figure 3. Downregulation of PVT1 inhibits cell proliferation, invasion, migration and promotes apoptosis in OSCC. (A) qPCR showed that PVT1 expression was inhibited in SCC-25 and CAL-27 cells transfected with LV-shPVT1 compared with the LV-sh NC group. (B and C) CCK-8 assay revealed that the proliferation abilities were suppressed in SCC-25 and CAL-27 cells transfected with LV-shPVT1 compared with the LV-sh NC group. (D-G) Transwell assays revealed that invasion and migration were suppressed in SCC-25 and CAL-27 cells transfected with LV-shPVT1 compared with the LV-sh NC group. (H-K) Protein expression levels of Ki67, Bcl-2, Bax, cleaved caspase 3, MMP-9 and MMP-14 were detected by western blot analysis in SCC-25 and CAL-27 cell lines transfected with LV-PVT1 when compared with the LV-NC group $\left({ }^{*} \mathrm{P}<0.05 ;{ }^{* * *} \mathrm{P}<0.01,{ }^{* * *} \mathrm{P}<0.001\right)$. PVT1, IncRNA plasmacytoma variant translocation 1; OSCC, oral squamous cell carcinoma; Bcl-2, B-cell lymphoma 2; Bax, bcl-2-like protein 4; MMP, matrix metalloproteinase; qPCR, real-time quantitative PCR.

MMP-9 and MMP-14 were inhibited following miR-150-5p overexpression (Fig. 5H-K). The results demonstrated that miR-150-5p regulated the biological functions in OSCC. As we know that miRNAs participate in various biological functions by inhibiting target genes, however, the detailed mechanism of miR-150-5p in OSCC remained unknown.

miR-150-5p negatively interacts with GLUT-1. To further explore the detailed mechanism of miR-150-5p in OSCC, downstream targets of miR-150-5p were analyzed using TargetScan database. As a result, GLUT-1 was predicted as a downstream target of miR-150-5p, which is associated with cell proliferation and tumorigenesis (37-39). We first detected the GLUT-1 protein expression in OSCC tumor tissues and adjacent non-tumor tissues. Western blot analysis revealed that the protein expression of GLUT-1 was increased 2.1-fold in the OSCC tumor tissues when compared with that in the adjacent tissues (Fig. 6A) $(\mathrm{P}<0.001)$. Furthermore, GLUT-1 
A
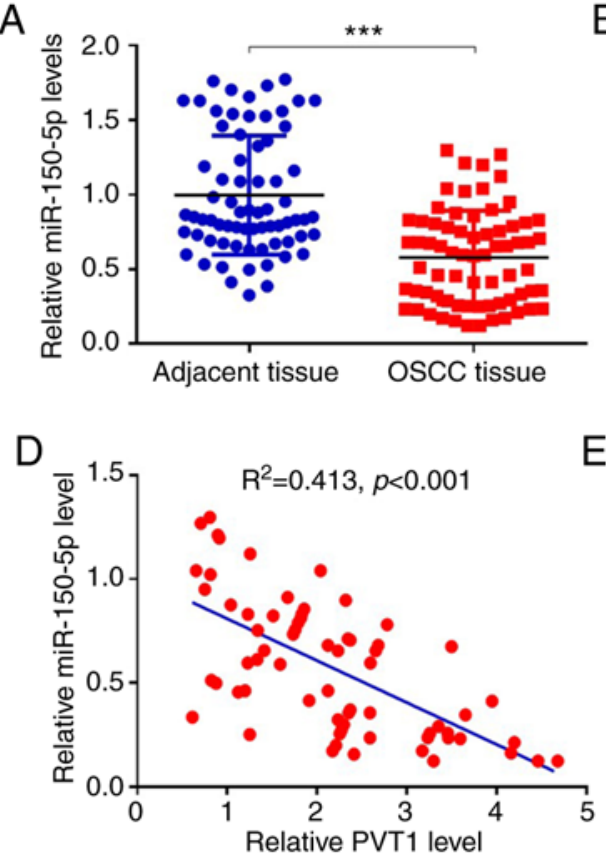

G

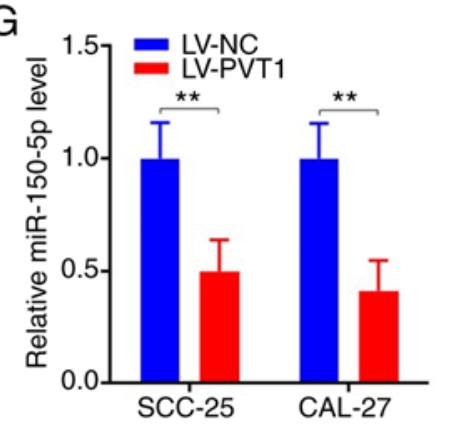

B

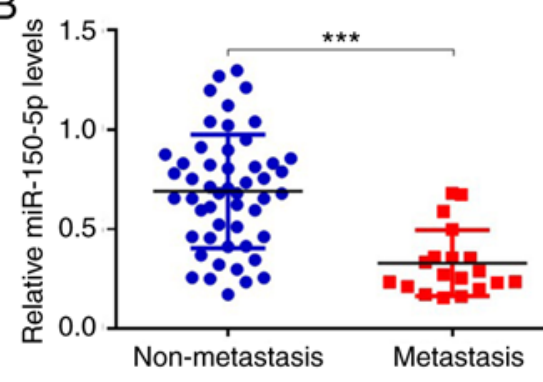

E

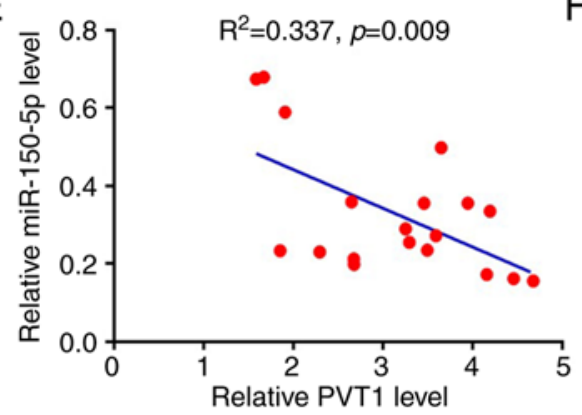

C

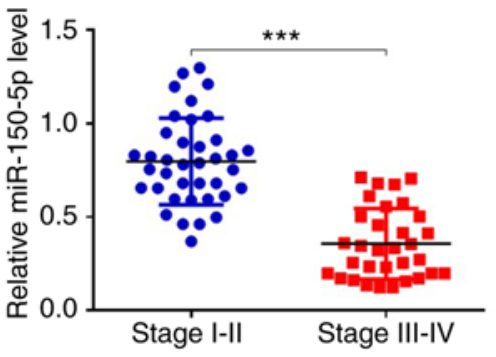

$\mathrm{F}$

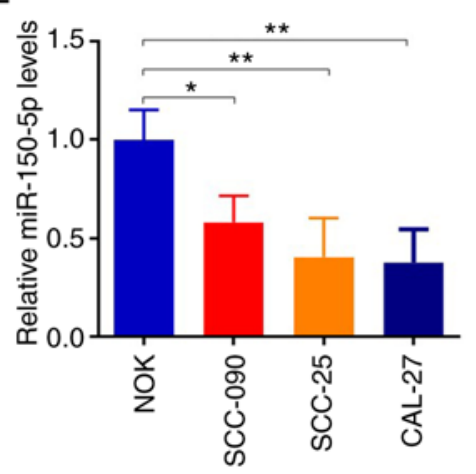

$\mathrm{H}$

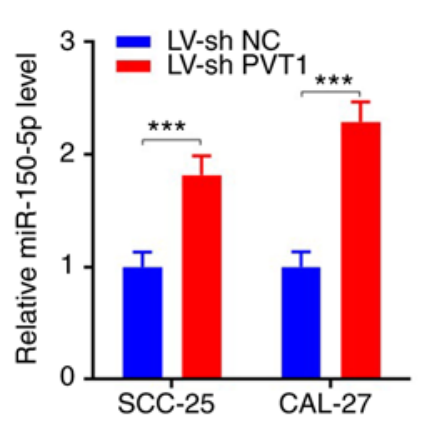

I

WT PVT1 5'-CAACGUGCUGGGAGAACA-3' miR-150-5p 3'-GUUCCCAACCCUCU-5

MUT PVT1 5'-CAACGUGCACCCUCCACA-3'
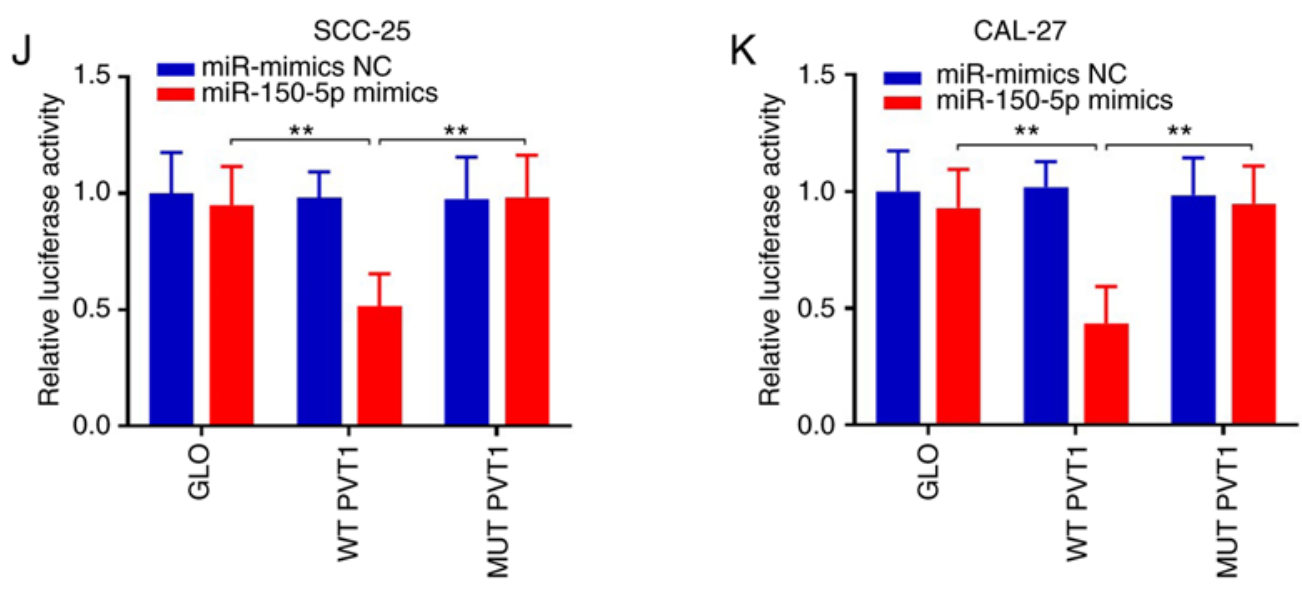

Figure 4. PVT1 directly sponges miR-150-5p in OSCC. (A) qPCR demonstrated that miR-150-5p was downregulated in OSCC tissues when compared with the adjacent tissues. (B) qPCR demonstrated that miR-150-5p was lower in patients with metastasis $(\mathrm{n}=19)$ than those with non-metastasis $(\mathrm{n}=51)$. $(\mathrm{C}) \mathrm{qPCR}$ indicated that miR-150-5p in patients at stage III-IV was much lower than that at stage I-II. (D and E) Correlation analysis demonstrated that miR-150-5p is negatively correlated with PVT1 in OSCC and metastasis patients. (F) qPCR demonstrated that miR-150-5p was decreased in OSCC cells lines when compared to human normal oral epithelial NOK cells. (G and H) miR-150-5p expression was determined in SCC-25 and CAL-27 cells transfected with LV-PVT1 and LV-sh PVT1 by qPCR. (I) Potential binding sequence and mutant sequence were constructed. (J and K) Luciferase gene reporter assays demonstrated that PVT1 could bind with miR-150-5p in OSCC $\left({ }^{*} \mathrm{P}<0.05 ;{ }^{* * *} \mathrm{P}<0.01,{ }^{* * *} \mathrm{P}<0.001\right)$. PVT1, lncRNA plasmacytoma variant translocation 1 ; OSCC, oral squamous cell carcinoma; qPCR, real-time quantitative PCR; WT, wild-type; MUT, mutant.

expression was increased 1.8 -fold in the OSCC tissues with metastasis $(n=19)$, compared to those without metastasis $(n=51)$ (Fig. 6B) $(\mathrm{P}<0.001)$. Moreover, protein expression of GLUT-1 in patients with stage III-IV were much higher than those at stage I-II (Fig. 6C) $(\mathrm{P}<0.001)$. We also found that miR-150-5p was negatively correlated with GLUT-1 in OSCC patients and metastasis patients (Fig. 6D and E). Additionally, PVT1 was positively correlated with GLUT-1 in OSCC 
A
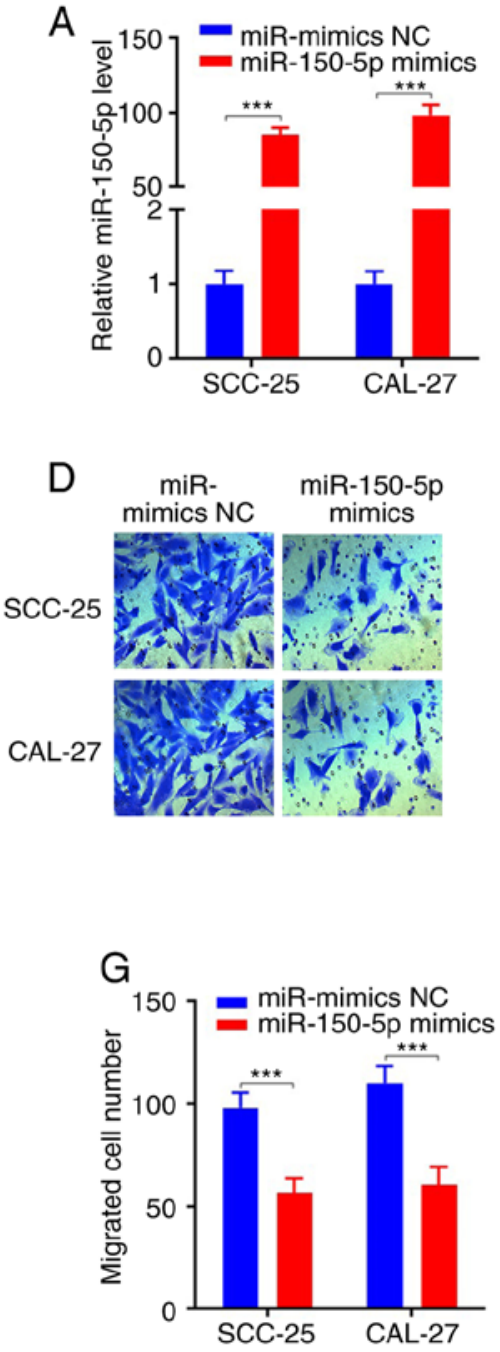

B

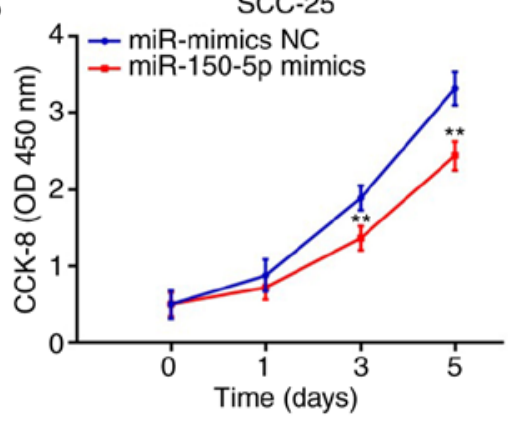

E

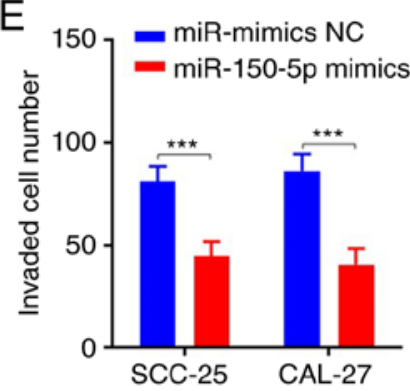

CAL-27

C

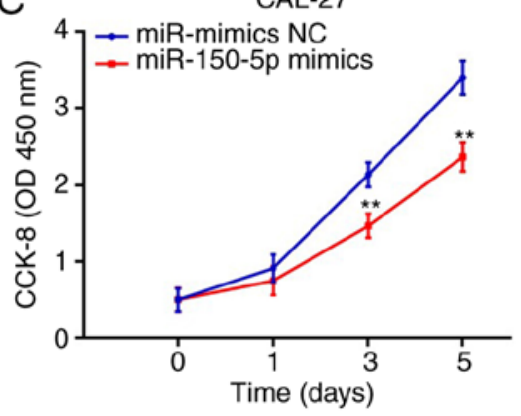

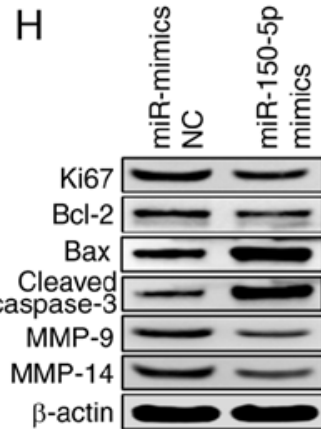
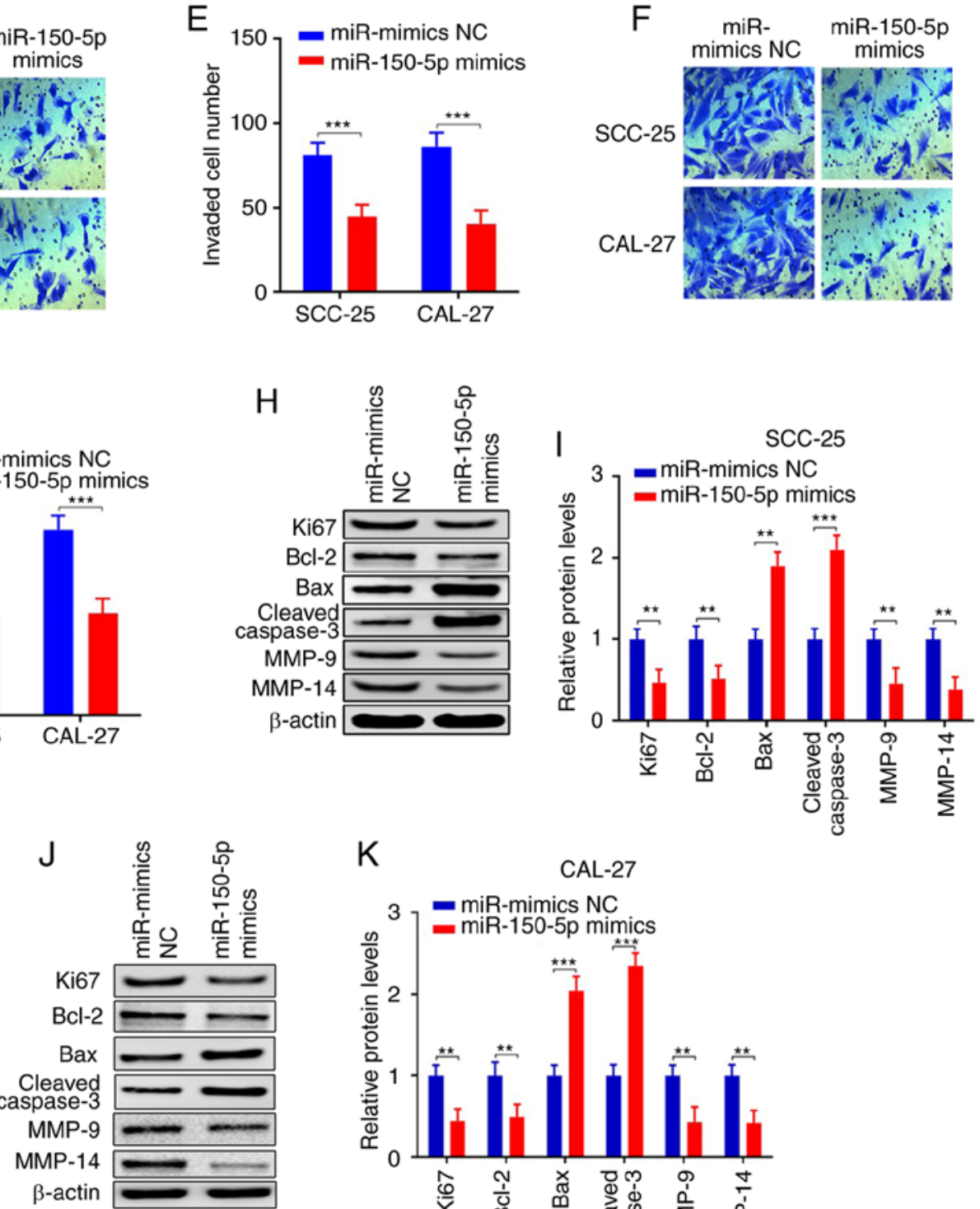

$\mathrm{K}$

CAL-27

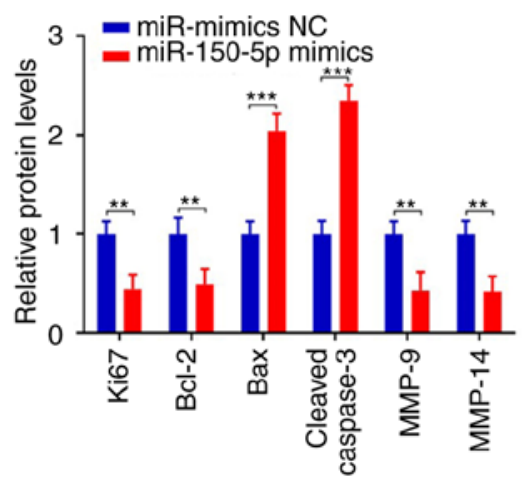

Figure 5. miR-150-5p inhibits cell proliferation, invasion, migration and promotes apoptosis in OSCC. (A) qPCR demonstrate that miR-150-5p was upregulated after miR-150-5p mimic transfection in the SCC-25 and CAL-27 cell lines when compared with the miR-mimics NC group. (B and C) CCK-8 assays revealed that the proliferation abilities were inhibited in the SCC-25 and CAL-27 cell lines transfected with the miR-150-5p mimics when compared with the miR-mimics NC group. (D-G) Transwell assays revealed that invasion and migration were inhibited in the SCC-25 and CAL-27 cell lines transfected with the miR-150-5p mimics when compared with the miR-mimics NC group. (H-K) Protein expression levels of Ki67, Bcl-2, Bax, cleaved caspase 3, MMP-9 and MMP-14 were detected by western blot analysis in the SCC-25 and CAL-27 cell lines transfected with the miR-150-5p mimics when compared with the miR-mimics $\mathrm{NC}$ group $\left({ }^{* *} \mathrm{P}<0.01,{ }^{* * *} \mathrm{P}<0.001\right)$. OSCC, oral squamous cell carcinoma; Bcl-2, B-cell lymphoma 2; Bax, bcl-2-like protein 4; MMP, matrix metalloproteinase; qPCR, real-time quantitative PCR.

patients and metastasis patients (Fig. 6F and G). In addition, protein expression of GLUT-1 was increased in OSCC cell lines compared with that in the NOK cells (Fig. 6H). Finally, expression of GLUT-1 was suppressed in SCC-25 and CAL-27 
A
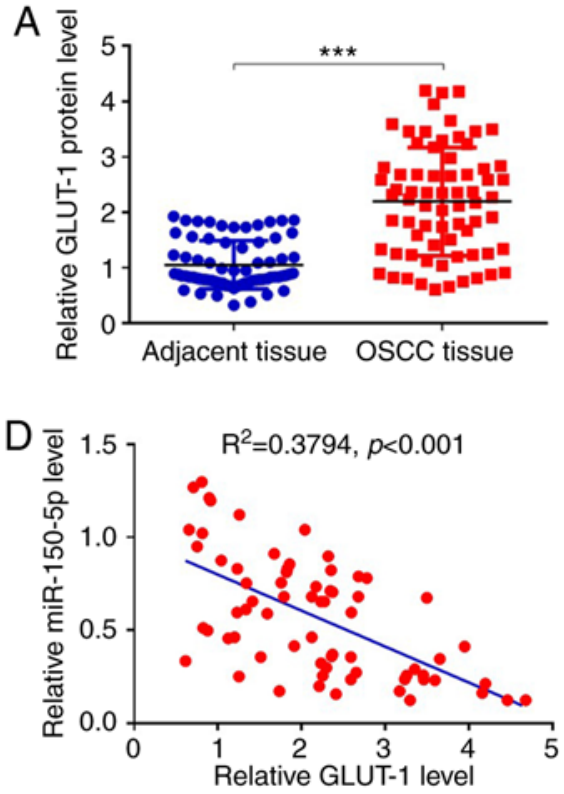

G

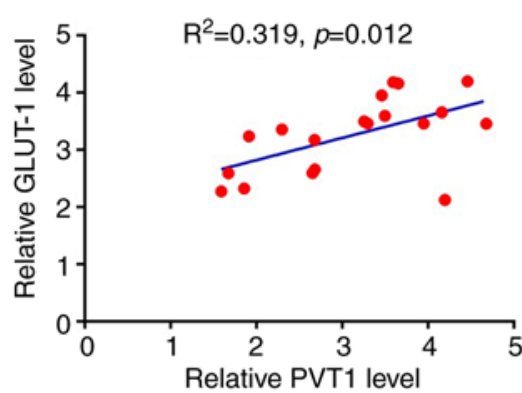
WT GLUT-1
5'-GACCAGUUGGGAGC-3'
miR-150-5p
3'-GUUCCCAACCCUCU-5'
MUT GLUT-1
5'-GACCACAACCCUCC-3'
$\mathrm{B}$

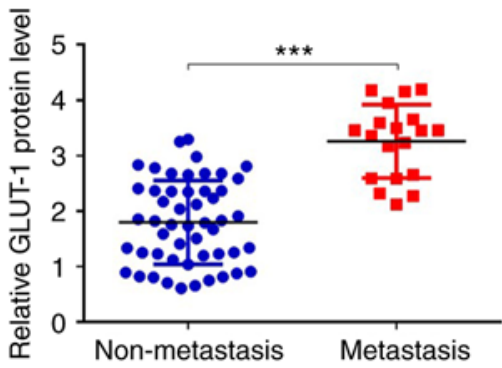

$\mathrm{E} \underset{\mathrm{d}}{\Phi}$

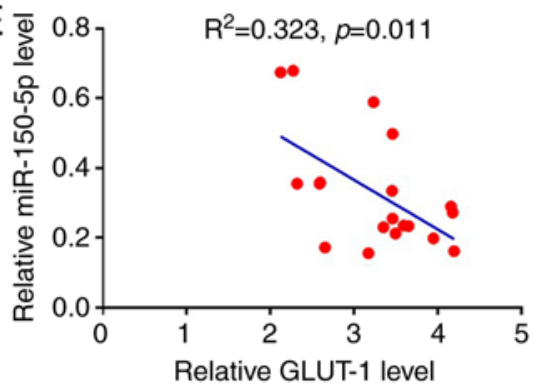

$\mathrm{H}$

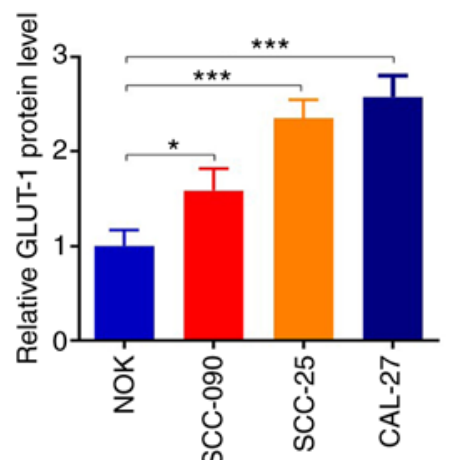

SCC- 25

$\mathrm{K}$

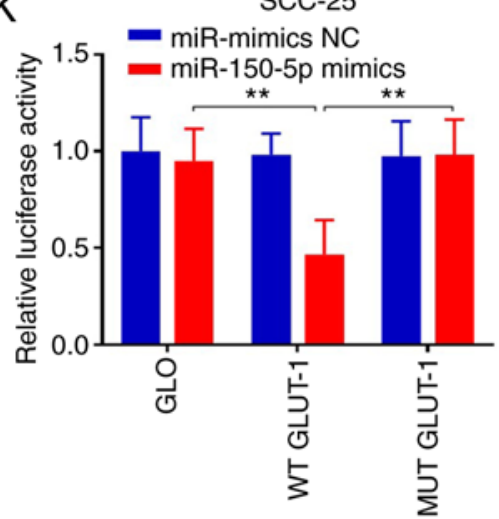

C
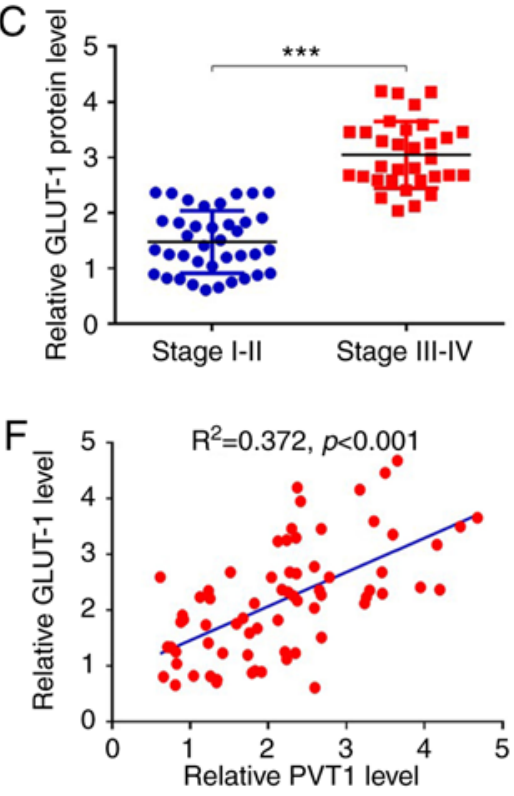

I $\bar{\Phi} 15=$ miR-mimics NC

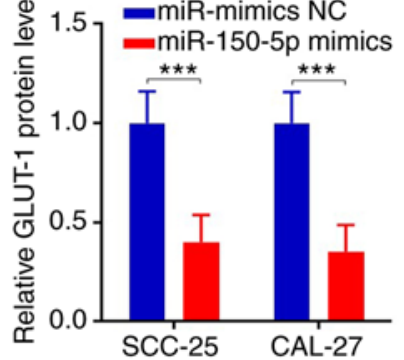

$\mathrm{L}$

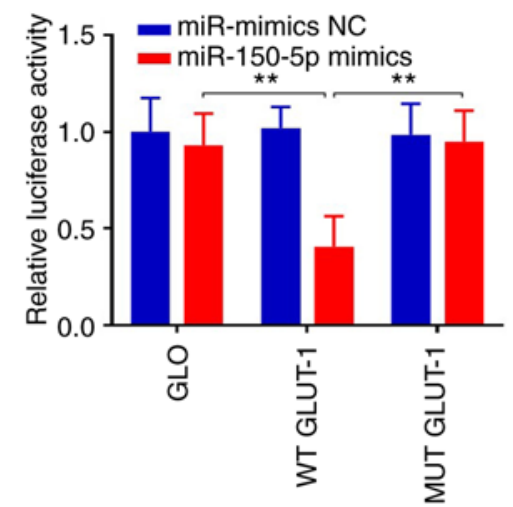

Figure 6. miR-150-5p is negatively correlated with GLUT-1. (A) qPCR demonstrated that GLUT-1 was increased 2.1-fold in OSCC tissues when compared with the adjacent tissues. (B) qPCR revealed that GLUT-1 expression was increased 1.8-fold in OSCC tissues with metastasis when compared with tissues without metastasis $(\mathrm{n}=19)$. (C) qPCR indicated that GLUT-1 protein expression in patients at stage III-IV was higher than that in stage I-II. (D and E) miR-150-5p is negatively correlated with GLUT-1 in OSCC and metastasis patients. (F and G) Correlation analysis indicated that PVT1 is positively correlated with GLUT-1 in OSCC and metastasis patients. (H) qPCR revealed that GLUT-1 is increased in OSCC cell lines when compared to human normal oral epithelial NOK cells. (I) Expression of GLUT-1 was suppressed after miR-150-5p mimic transfection in the SCC-25 and CAL-27 cell lines compared with the miR-mimics NC group. (J) Potential binding sequence and mutant sequence were constructed. (K and L) miR-150-5p directly binds to GLUT-1 ( $\left(\mathrm{P}<0.05 ;{ }^{* *} \mathrm{P}<0.01,{ }^{* * *} \mathrm{P}<0.001\right)$. GLUT1, glucose transporter 1; PVT1, IncRNA plasmacytoma variant translocation 1; OSCC, oral squamous cell carcinoma; qPCR, real-time quantitative PCR; WT, wild-type; MUT, mutant.

cells following miR-150-5p overexpression (Fig. 6I) $(\mathrm{P}<0.001)$. Above all, miR-150-5p was negatively correlated with GLUT-1, which may be a potential target for miR-150-5p.

To confirm whether miR-150-5p targets GLUT-1 in OSCC, WT-GLUT-1 and MUT-GLUT-1 sequences were constructed into vectors and the luciferase gene reporter assay was performed (Fig. 6J). The results demonstrated that miR-150-5p overexpression attenuated luciferase activity of WT-GLUT-1 but not of MUT-GLUT-1 (Fig. 6K and L) $(\mathrm{P}<0.01)$. The above data showed that miR-150-5p could inhibit GLUT-1 and it 

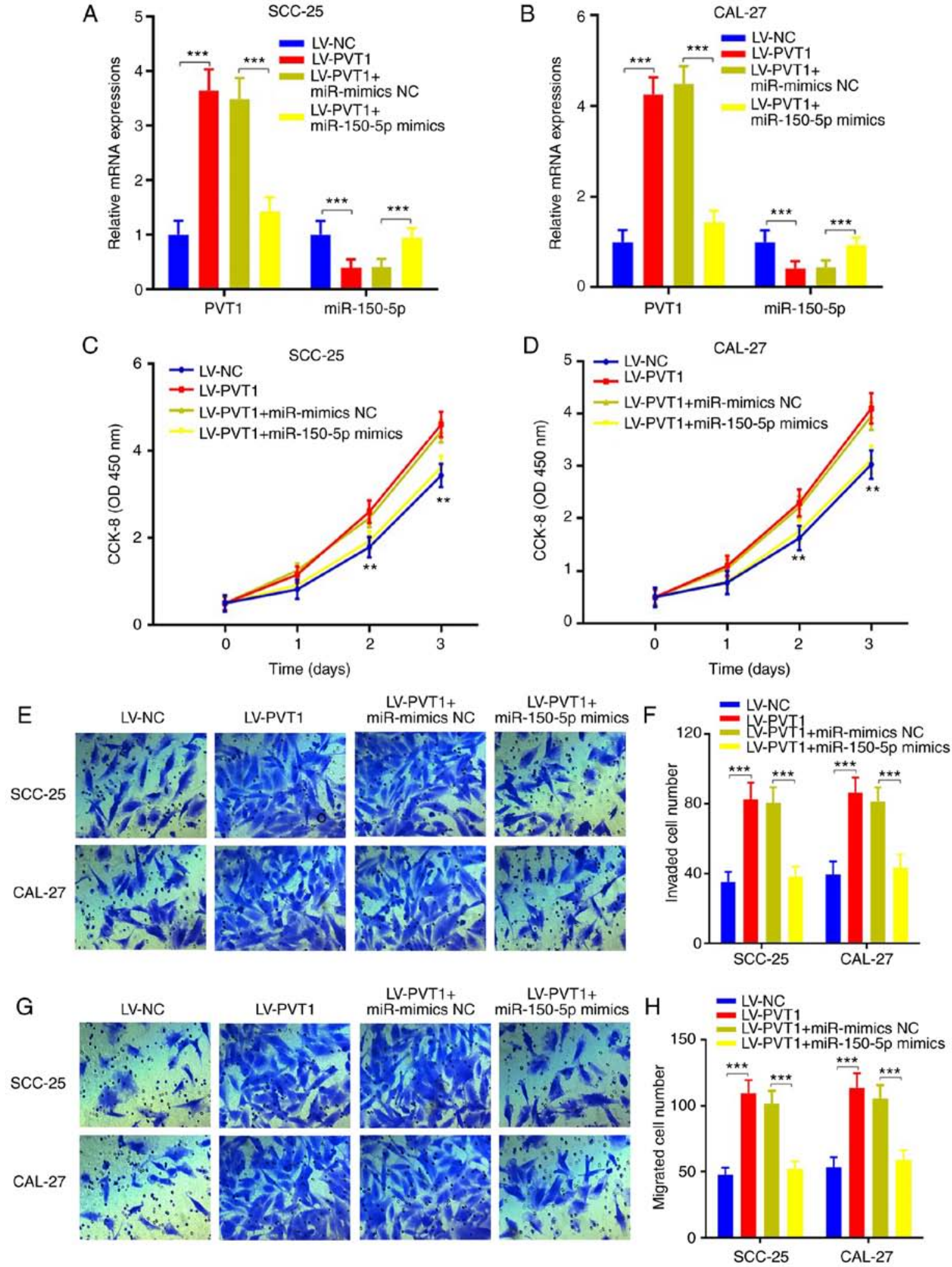

Figure 7. PVT1 promotes cell proliferation, invasion, migration and inhibits apoptosis via the miR-150-5p/GLUT-1 axis in patients with OSCC. (A and B) Expression of PVT1 and miR-150-5p were detected in SCC-25 and CAL-27 cell lines following the indicated transfections. (C and D) CCK-8 assay indicated that the LV-PVT1-promoted cell proliferation abilities were suppressed following miR-150-5p overexpression. (E-H) The LV-PVT1-promoted increases in cell invasion and migration were suppressed following miR-150-5p mimic transfection. $\left({ }^{* *} \mathrm{P}<0.01,{ }^{* * *} \mathrm{P}<0.001\right)$. GLUT1, glucose transporter 1 ; PVT1, IncRNA plasmacytoma variant translocation 1; OSCC, oral squamous cell carcinoma.

may be a target of miR-150-5p in OSCC. Overall, PVT1 may directly bind with miR-150-5p, which targets GLUT-1 expression, thereby affecting biological functions in patients with OSCC.
PVT1 promotes cell proliferation, invasion, migration and inhibits apoptosis via the miR-150-5p/GLUT-1 axis in patients with OSCC. To confirm our hypothesis, miR-150-5p mimics or miR-NC was respectively transfected into cells infected with 

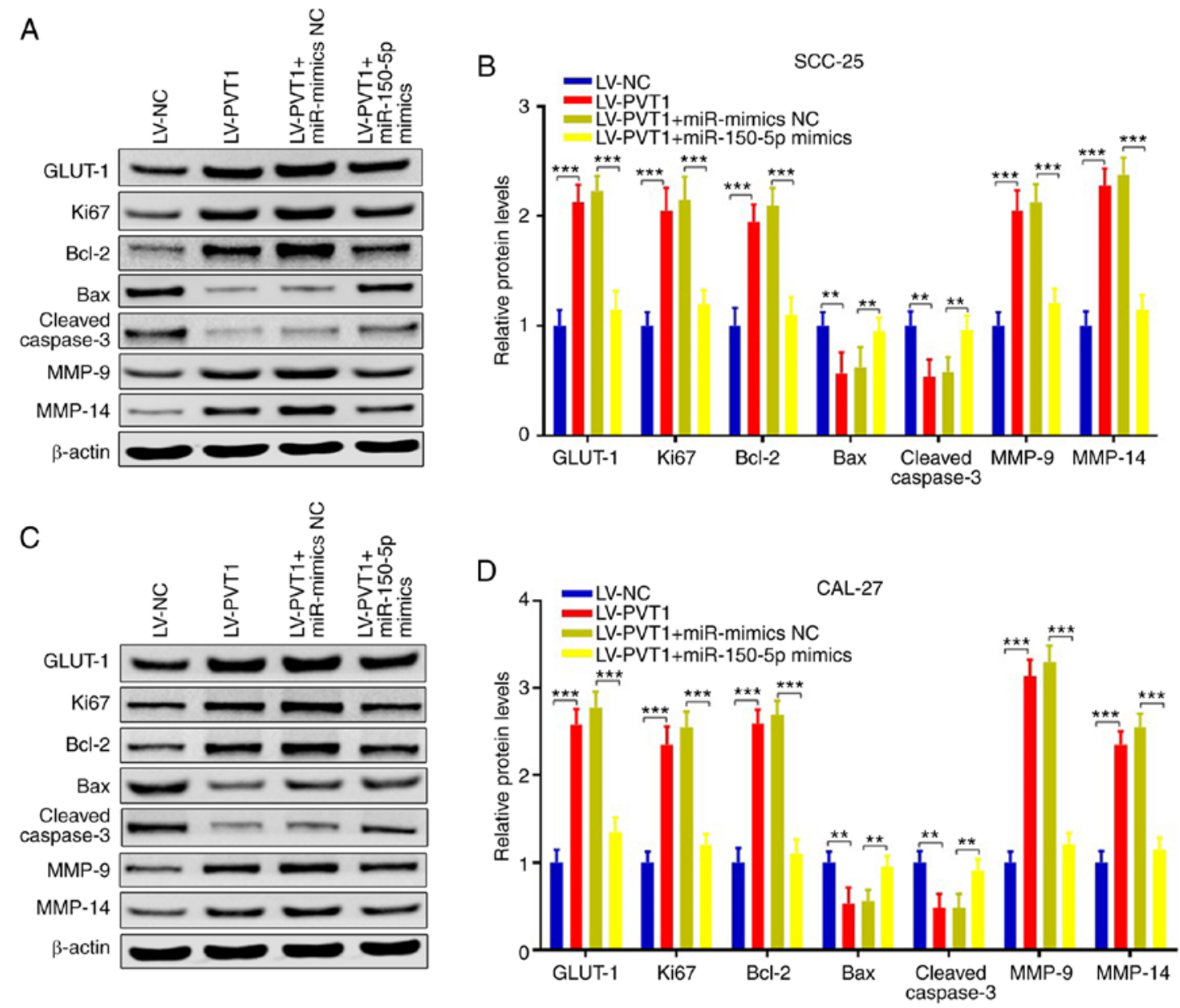

Figure 8. PVT1 regulates the expression of proteins associated with proliferation, invasion, apoptosis and migration via the miR-150-5p/GLUT-1 axis in patients with OSCC. (A-D) Western blot analyses showed that protein levels of GLUT-1, Ki67, Bcl-2, Bax, cleaved caspase 3, MMP-9 and MMP-14 were consistent with our previous results, while they were reversed following miR-150-5p overexpression. $\left.{ }^{* *} \mathrm{P}<0.01,{ }^{* * *} \mathrm{P}<0.001\right)$. GLUT1, glucose transporter 1 ; PVT1, lncRNA plasmacytoma variant translocation 1; OSCC, oral squamous cell carcinoma; Bcl-2, B-cell lymphoma 2; Bax, bcl-2-like protein 4; MMP, matrix metalloproteinase.

LV-PVT1, and cell proliferation ability, invasive and migratory abilities were evaluated. The results showed that PVT1 was increased andmiR-150-5pwasrepressedinLV-PVT1 transfected cells, while PVT1 was reduced and miR-150-5p was increased following miR-150-5p overexpression in the SCC-25 and CAL-27 cells (Fig. 7A and B) $(\mathrm{P}<0.001)$. Furthermore, CCK-8 assays indicated that the LV-PVT1-overexpression-enhanced cell proliferation abilities were suppressed following miR-150-5p overexpression (Fig. 7C and D) $(\mathrm{P}<0.01)$. Moreover, the LV-PVT1-overexpression-enhanced cell invasion and migration were suppressed following miR-150-5p overexpression (Fig. 7E-H) $(\mathrm{P}<0.001)$. In addition, we also detected the gene expression of GLUT-1, Ki67, Bcl-2, Bax, cleaved caspase 3, MMP-9 and MMP-14. The results showed that these protein levels were consistent with our previous results, while they were reversed following by miR-150-5p overexpression in the SCC-25 (Fig. 8A and B) and CAL-27 cell line (Fig. 8C and D). Collectively, PVT1 promotes cell proliferation, invasion, migration and inhibits apoptosis via the miR-150-5p/GLUT-1 axis in patients with OSCC.

PVT1 inhibition suppresses tumor growth and expression of invasion and migration-associated genes in vivo. To verify the functions of PVT1 in vivo, we injected SCC-25 cells that had been stably infected with LV-shPVT1 or LV-sh NC into nude mice. Tumor volumes were measured every week until the 5th week. Tumors infected with LV-shPVT1 showed smaller tumor volume (Fig. 9A). The tumor volumes in the LV-shPVT1 group were significantly smaller at day 28 and 35 (Fig. 9B) $(\mathrm{P}<0.001)$. At day 35, the mean value of the orthotopic tumor weight of LV-shPVT1 tumors was lighter than that noted in the LV-sh NC group (Fig. 9C) $(\mathrm{P}<0.001)$. Furthermore, we detected expression levels of PVT1, miR-150-5p, GLUT-1, Ki67, Bcl-2, Bax, cleaved caspase3, MMP-9 and MMP-14 in the tumors derived from cells transfected with LV-shNC or LV-sh PVT1. The results showed that PVT1 and GLUT-1 were decreased in the LV-shPVT1 group, while miR-150-5p was increased (Fig. 9D) $(\mathrm{P}<0.001)$. The protein expression levels of $\mathrm{Ki} 67$ and $\mathrm{Bcl}-2$ were decreased, while Bax and cleaved caspase 3 were increased, which suggested that the proliferation ability was suppressed in the LV-shPVT1 group. The protein expression levels of EMT markers, including GLUT-1, MMP-9 and MMP-14, were reduced in the LV-shPVT1 group, indicating that the invasive and migrated abilities were inhibited (Fig. 9E and F). These results confirmed that PVT1 is critical for tumor growth, invasion and migration via the miR-150-5p/GLUT-1 pathway in human OSCC. 

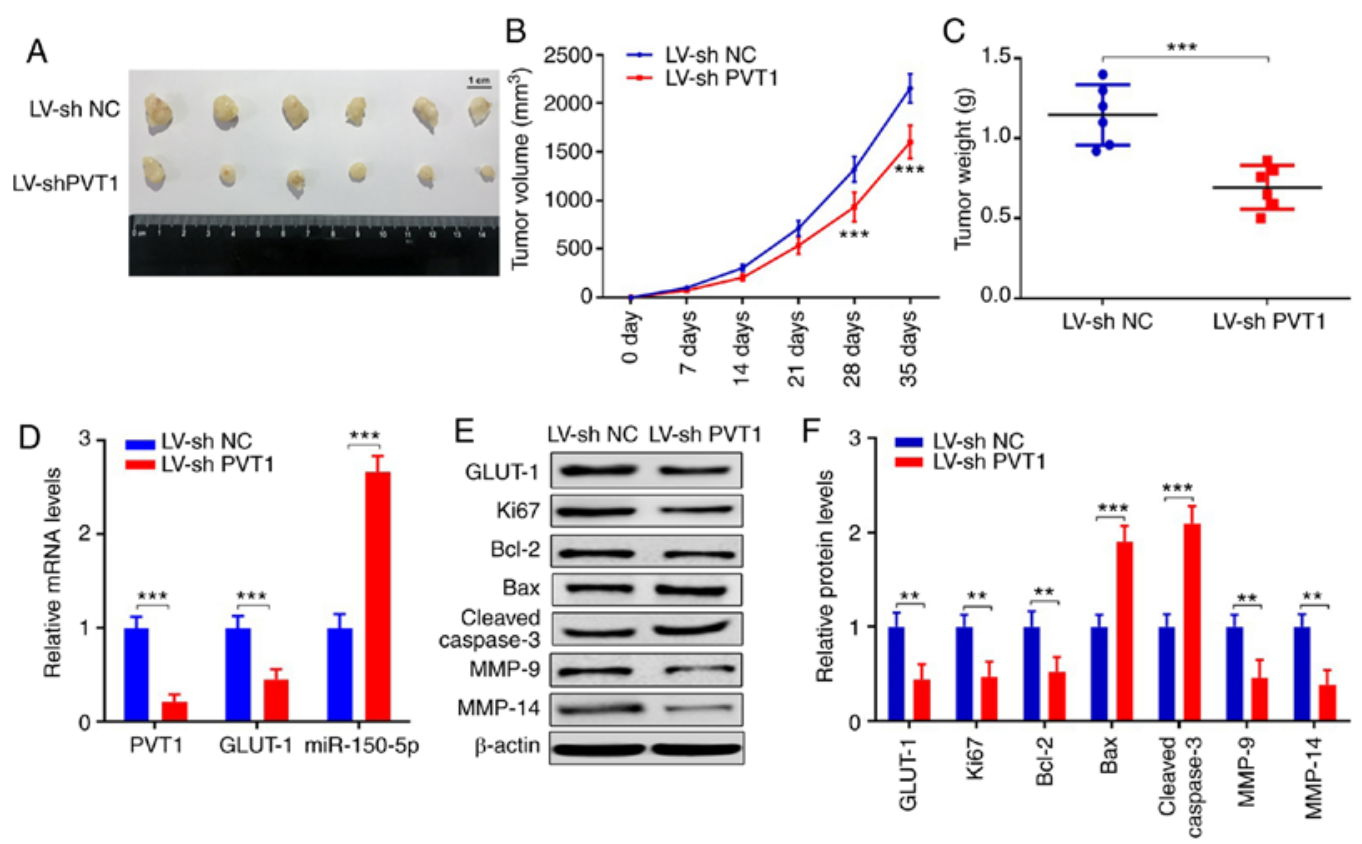

Figure 9. PVT1 inhibition suppressed tumor growth and expression of proteins associated with invasion and migration in vivo. (A) Images of 12 primary tumors at day 35 from the LV-shPVT1 and LV-sh NC groups. (B) Tumor volumes were measured every week for 35 days. (C) Tumor weights at day 35 after injection were calculated. (D) mRNA levels of PVT1, miR-150-5p, GLUT-1 were detected in the tumor tissues of the LV-sh NC and LV-sh PVT1 groups. (E and F) Protein expression levels of GLUT-1, Ki67, Bcl-2, Bax, cleaved caspase 3, MMP-9 and MMP-14 were detected by western blot analysis ("* P $<0.01$, $\left.{ }^{* * *} \mathrm{P}<0.001\right)$. GLUT1, glucose transporter 1; PVT1, IncRNA plasmacytoma variant translocation 1; OSCC, oral squamous cell carcinoma; Bcl-2, B-cell lymphoma 2; Bax, bcl-2-like protein 4; MMP, matrix metalloproteinase.

\section{Discussion}

Abnormal expression of long non-coding RNAs (lncRNAs) and microRNAs (miRNAs/miRs) has been demonstrated to be related to tumor formation and progression in various types of cancers (10-12). For example, lncRNA HOTAIR was found to be suppressed in ovarian cancer cells, which was found to inhibit the invasion and tumorigenicity by interacting with miR-200c in mice (11). lncRNA MIR4435-2HG was found to promote prostate cancer migration and invasion by upregulating TGF- $\beta 1$ expression (10). LINC00511 was upregulated in estrogen receptor (ER)-negative breast cancer, which was associated with poor prognosis for patients and it could act as an oncogene by interacting with EZH2 and PRC2 (12). Recently, increasing evidence has revealed that lncRNAs play oncogenic or tumor-suppressor roles in oral squamous cell carcinoma (OSCC) (13-15). IncRNA plasmacytoma variant translocation 1 (PVT1) was confirmed to participate in the promotion of tumor formation, invasion and migration in many types of cancers, such as glioma, colorectal, gastric, ovarian, gallbladder and breast cancer (16-21). However, the functions of PVT1 in OSCC remains unclear.

In the present study, we found that PVT1 was upregulated in OSCC tissues and cell lines, and was associated with metastasis, advanced stages and poor overall patient survival. The results revealed that PVT1 overexpression promoted cell proliferation, invasion, migration and apoptosis, while PVT1 downregulation produced the opposite results, which suggests that PVT1 is an oncogenic gene in OSCC. This finding was consistent with former reports in various types of cancers, such as glioma, colorectal, gastric, ovarian, gallbladder and breast cancer (16-21). However, the underlying mechanisms of PVT1 in tumorigenesis and migration in OSCC remain unknown.
miRNAs act as oncogenes or tumor suppressors in cancers, and have been reported to be target genes of IncRNAs $(27,28)$. We used Starbase v2.0 database which indicated that miR-150-5p may be a downstream target of PVT1. We also uncovered that miR-150-5p expression was reduced in OSCC tumor tissues, and was negatively correlated with PVT1. In order to determine the interaction between PVT1 and miR-150-5p, dual-luciferase gene reporter assay was performed and the results demonstrated that miR-150-5p overexpression attenuated luciferase activity in the wild-type WT-PVT1 but not mutant MUT-PVT1, which indicated that PVT1 could function as a competing endogenous RNA (ceRNA), to competitively bind to miR-150-5p in OSCC. Moreover, miR-150-5p overexpression inhibited cell proliferation, invasion and migration in OSCC. Collectively, PVT1 serves as an oncogenic gene in OSCC via binding with miR-150-5p, which are important for cancer biology; however, the underlying mechanism remained unknown.

Glucose transporter type 1 (GLUT1) is an oncogenic gene that is associated with the biological functions and progression of various types of cancers, such as bladder, non-small cell lung and prostate cancer (37-39), and may be a downstream target for miR-150-5p. GLUT1 is a glucose membrane transporter that can uptake glucose and regulate the metabolism in cancers through several mechanisms $(42,43)$. Firstly, transported glucose will follow the glycolytic pathway to generate pyruvate, which is converted into lactate under the condition of anaerobiosis and it will supply energy for cancer cells $(44,45)$. Secondly, pyruvate can be transformed into acetyl-coenzyme-A (Acetyl-CoA), which can be supplied to ATP generation and promote cancer cell proliferation (46-48). Finally, cancer cells can use substrates from glucose as a carbon source, such as fatty acids and glutamine $(49,50)$. 
In the present study, we found that GLUT1 protein expression was increased in OSCC tumor tissues, and was negatively correlated with miR-150-5p. Luciferase assay indicated that miR-150-5p overexpression attenuated luciferase activity in WT-GLUT-1 but not MUT-GLUT-1, which suggested that miR-150-5p could directly bind with GLUT-1 in OSCC. Collectively, we assumed that PVT1 could directly bind with miR-150-5p, which attenuated GLUT-1 inhibition, thereby promoting cell proliferation, invasion and migration in OSCC. Collectively, we found that overexpression of PVT1 promoted cell proliferation, invasion and migration of OSCC via targeting the miR-150-5p/GLUT-1 pathway.

To verify the functions of PVT1 in vivo, a nude mouse xenograft model was performed and SCC-25 cells stably transfected with LV-shPVT1 or LV-NC were injected into the mice. The results revealed that the tumor volumes and tumor weight were reduced in the LV-shPVT1 group when compared with the LV-NC group. Furthermore, GLUT-1 was decreased and miR-150-5p was upregulated in the LV-shPVT1 group. In addition, the protein expression levels of Ki67, Bcl-2, GLUT-1, MMP-9 and MMP-14 were decreased, while Bax and cleaved caspase 3 were increased in the LV-shPVT1 group, suggesting that the proliferation, invasion and migration capacities were suppressed in the LV-shPVT1 group in vivo.

In the present study, we performed in situ hybridization, but the results showed poor specificity and the quantification was not precise, which was a limitation of this present study and we will improve the in situ hybridization experiment in future research. On the other hand, we used RT-qPCR to detect PVT1 expressions in OSCC tissues and adjacent tissues, which showed a very high specificity and quantification. According to the results of the RT-qPCR, we carried out further research to explore the functions of PVT1, and we found that the elevated PVT1 promoted tumor cell proliferation, invasion, migration and inhibited apoptosis in the OSCC cells.

In conclusion, the present study revealed that PVT1 is increased in OSCC tissues, and is associated with the poor prognosis of OSCC patients. We uncovered a previously unappreciated PVT1/miR-150-5p/GLUT-1 signaling axis in promoting cell proliferation, invasion and migration in OSCC cells in vitro and also in vivo, which suggests that the PVT1/miR-150-5p/GLUT-1 signaling axis may be a target for the treatment of OSCC.

\section{Acknowledgements}

Not applicable.

\section{Funding}

No funding was received.

\section{Availability of data and materials}

The datasets used during the present study are available from the corresponding author upon reasonable request.

\section{Authors' contributions}

XL and HR conceived and designed the study. XL performed the experiments and wrote the manuscript. HR reviewed and edited the manuscript. Both authors read and approved the manuscript and agree to be accountable for all aspects of the research in ensuring that the accuracy or integrity of any part of the work are appropriately investigated and resolved.

\section{Ethics approval and consent to participate}

The protocol for collected the patient samples were approved by the Faculty of Medicine's Ethics Committee of The First Affiliated Hospital of Jinzhou Medical University (ethical approval no. JYD160923). All animal handling and experimental procedures were approved by the Animal Ethics Committees of the First Affiliated Hospital of Jinzhou Medical University and in accordance with the guidelines of the China Council of Animal Care.

\section{Patient consent for publication}

Not applicable.

\section{Competing interests}

The authors declare that they have no competing interests.

\section{References}

1. Wangmo C, Charoen N, Jantharapattana K, Dechaphunkul A and Thongsuksai P: Epithelial-mesenchymal transition predicts survival in oral squamous cell carcinoma. Pathol Oncol Res 26: 1511-1518, 2020.

2. Huang F, Xin C, Lei K, Bai H, Li J and Chen Q: Noncoding RNAs in oral premalignant disorders and oral squamous cell carcinoma. Cell Oncol (Dordr): June 3, 2020 (Epub ahead of print).

3. Gharat SA, Momin M and Bhavsar C: Oral squamous cell carcinoma: Current treatment strategies and nanotechnology-based approaches for prevention and therapy. Crit Rev Ther Drug Carrier Syst 33: 363-400, 2016.

4. Almangush A, Mäkitie AA, Triantafyllou A, de Bree R, Strojan P, Rinaldo A, Hernandez-Prera JC, Suárez C, Kowalski LP Ferlito A and Leivo I: Staging and grading of oral squamous cell carcinoma: An update. Oral Oncol 107: 104799, 2020.

5. Warnakulasuriya S: Global epidemiology of oral and oropharyngeal cancer. Oral Oncol 45: 309-316, 2009.

6. Chen W, Zheng R, Baade PD, Zhang S, Zeng H, Bray F, Jemal A Yu XQ and He J: Cancer statistics in China, 2015. CA Cancer J Clin 66: 115-132, 2016.

7. Hauptman $\mathrm{N}$ and Glavac D: MicroRNAs and long non-coding RNAs: Prospects in diagnostics and therapy of cancer. Radiol Oncol 47: 311-318, 2013.

8. Morris KV and Mattick JS: The rise of regulatory RNA. Nat Rev Genet 15: 423-437, 2014.

9. Luo W, Wang M, Liu J, Cui X and Wang H: Identification of a six lncRNAs signature as novel diagnostic biomarkers for cervical cancer. J Cell Physiol 235: 993-1000, 2020.

10. Zhang H, Meng H, Huang X, Tong W, Liang X, Li J, Zhang C and Chen M: IncRNA MIR4435-2HG promotes cancer cell migration and invasion in prostate carcinoma by upregulating TGF- $\beta 1$. Oncol Lett 18: 4016-4021, 2019.

11. Yang C, Li H, Zhang T, Chu Y, Chen D and Zuo J: miR-200c overexpression inhibits the invasion and tumorigenicity of epithelial ovarian cancer cells by suppressing lncRNA HOTAIR in mice. J Cell Biochem 121: 1514-1523, 2020.

12. Zhang J, Sui S, Wu H, Zhang J, Zhang X, Xu S and Pang D: The transcriptional landscape of lncRNAs reveals the oncogenic function of LINC00511 in ER-negative breast cancer. Cell Death Dis 10: 599, 2019.

13. Zhu G, Wang S, Chen J, Wang Z, Liang X, Wang X, Jiang J, Lang $\mathrm{J}$ and Li L: Long noncoding RNA HAS2-AS1 mediates hypoxia-induced invasiveness of oral squamous cell carcinoma. Mol Carcinog 56: 2210-2222, 2017. 
14. Kong J, Sun W, Zhu W, Liu C, Zhang H and Wang H: Long noncoding RNA LINC01133 inhibits oral squamous cell carcinoma metastasis through a feedback regulation loop with GDF15. J Surg Oncol 118: 1326-1334, 2018.

15. Jin Z, Jiang S, Jian S and Shang Z: Long noncoding RNA MORT overexpression inhibits cancer cell proliferation in oral squamous cell carcinoma by downregulating ROCK1. J Cell Biochem: Feb 25, 2019 (Epub ahead of print).

16. Song T, Yan L, Cai K, Zhao T and Xu M: Downregulation of long noncoding RNA PVT1 attenuates paclitaxel resistance in glioma cells. Cancer Biomark 23: 447-453, 2018.

17. Shang AQ, Wang WW, Yang YB, Gu CZ, Ji P, Chen C, Zeng BJ, Wu JL, Lu WY, Sun ZJ and Li D: Knockdown of long noncoding RNA PVT1 suppresses cell proliferation and invasion of colorectal cancer via upregulation of microRNA-214-3p. Am J Physiol Gastrointest Liver Physiol 317: G222-G232, 2019.

18. Zhao J, Du P, Cui P, Qin Y, Hu C, Wu J, Zhou Z, Zhang W, Qin L and Huang G: LncRNA PVT1 promotes angiogenesis via activating the STAT3/VEGFA axis in gastric cancer. Oncogene 37: 4094-4109, 2018.

19. Yang Q, Yu Y, Sun Z and Pan Y: Long non-coding RNA PVT1 promotes cell proliferation and invasion through regulating miR-133a in ovarian cancer. Biomed Pharmacother 106: 61-67, 2018.

20. Chen J, Yu Y, Li H, Hu Q, Chen X, He Y, Xue C, Ren F, Ren Z, Li J, et al: Long non-coding RNA PVT1 promotes tumor progression by regulating the miR-143/HK2 axis in gallbladder cancer. Mol Cancer 18: 33, 2019.

21. Yan C, Chen Y, Kong W, Fu L, Liu Y, Yao Q and Yuan Y: PVT1-derived miR-1207-5p promotes breast cancer cell growth by targeting STAT6. Cancer Sci 108: 868-876, 2017.

22. Dejene SB, Ohman AW, Du W, Randhawa D, Bradley A, Yadav N, Elias KM, Dinulescu DM and Setlur SR: Defining fallopian tube-derived miRNA cancer signatures. Cancer Med 8: 6709-6716, 2019.

23. Zhong G, Lou W, Yao M, Du C, Wei H and Fu P: Identification of novel mRNA-miRNA-lncRNA competing endogenous RNA network associated with prognosis of breast cancer. Epigenomics 11: 1501-1518, 2019.

24. Shukla GC, Singh J and Barik S: MicroRNAs: Processing, maturation, target recognition and regulatory functions. Mol Cell Pharmacol 3: 83-92, 2011.

25. Bartel DP: MicroRNAs: Target recognition and regulatory functions. Cell 136: 215-233, 2009.

26. Khan AQ, Ahmed EI, Elareer NR, Junejo K, Steinhoff M and Uddin S: Role of miRNA-regulated cancer stem cells in the pathogenesis of human malignancies. Cells 8: 840, 2019.

27. Salmena L, Poliseno L, Tay Y, Kats L and Pandolfi PP: A ceRNA hypothesis: The Rosetta Stone of a hidden RNA language? Cell 146: 353-358, 2011

28. Tay Y, Kats L, Salmena L, Weiss D, Tan SM, Ala U, Karreth F, Poliseno L, Provero P, Di Cunto F, et al: Coding-independent regulation of the tumor suppressor PTEN by competing endogenous mRNAs. Cell 147: 344-357, 2011.

29. Zhang S, Liao K, Miao Z, Wang Q, Miao Y, Guo Z, Qiu Y, Chen B, Ren L, Wei Z, et al: CircFOXO3 promotes glioblastoma progression by acting as a competing endogenous RNA for NFAT5, Neuro-oncol 21: 1284-1296, 2019.

30. Wang H, Sha L, Huang L, Yang S, Zhou Q, Luo X and Shi B: LINC00261 functions as a competing endogenous RNA to regulate BCL2L11 expression by sponging miR-132-3p in endometriosis. Am J Transl Res 11: 2269-2279, 2019.

31. Cao C, Xu Y, Du K, Mi C, Yang C, Xiang L, Xie Y and Liu W: LINC01303 functions as a competing endogenous RNA to regulate EZH2 expression by sponging miR-101-3p in gastric cancer. J Cell Mol Med 23: 7342-7348, 2019.

32. Yan J, Jia Y, Chen H, Chen W and Zhou X: Long non-coding RNA PXN-AS1 suppresses pancreatic cancer progression by acting as a competing endogenous RNA of miR-3064 to upregulate PIP4K2B expression. J Exp Clin Cancer Res 38: 390, 2019.
33. Zhang S, Dong X, Ji T, Chen G and Shan L: Long non-coding RNA UCA1 promotes cell progression by acting as a competing endogenous RNA of ATF2 in prostate cancer. Am J Transl Res 9: 366-375, 2017.

34. Lu W, Zhang H, Niu Y, Wu Y, Sun W, Li H, Kong J, Ding K, Shen HM, Wu H, et al: Long non-coding RNA linc00673 regulated non-small cell lung cancer proliferation, migration, invasion and epithelial mesenchymal transition by sponging miR-150-5p. Mol Cancer 16: 118, 2017.

35. Dai FQ, Li CR, Fan XQ, Tan L, Wang RT and Jin H: miR-150-5p inhibits non-small-cell lung cancer metastasis and recurrence by targeting HMGA 2 and $\beta$-catenin signaling. Mol Ther Nucleic Acids 16: 675-685, 2019.

36. Chen X, Xu X, Pan B, Zeng K, Xu M, Liu X, He B, Pan Y, Sun H and Wang S: miR-150-5p suppresses tumor progression by targeting VEGFA in colorectal cancer. Aging (Albany NY) 10: 3421-3437, 2018.

37. Al-Maghrabi JA, Qureshi IA and Khabaz MN: Immunhistochemical expression of GLUT1 is associated with low grade and low stage of urinary bladder cancer. Int J Clin Exp Pathol 12: 3049-3057, 2019.

38. Lee SY and Park JY: GLUT1 variants for predicting prognosis after surgery in non-small cell lung cancer. Ann Surg Oncol 25 (Suppl 3): S948-S949, 2018

39. Xiao H, Wang J, Yan W, Cui Y, Chen Z, Gao X, Wen X and Chen J: GLUT1 regulates cell glycolysis and proliferation in prostate cancer. Prostate 78: 86-94, 2018.

40. Livak KJ and Schmittgen TD: Analysis of relative gene expression data using real-time quantitative PCR and the 2(-Delta Delta C(T)) method. Methods 25: 402-408, 2001.

41. Li JH, Liu S, Zhou H, Qu LH and Yang JH: starBase v2.0 Decoding miRNA-ceRNA, miRNA-ncRNA and protein-RNA interaction networks from large-scale CLIP-Seq data. Nucleic Acids Res 42 (Database Issue): D92-D97, 2014

42. Mueckler M and Thorens B: The SLC2 (GLUT) family of membrane transporters. Mol Aspects Med 34: 121-138, 2013.

43. Deng D and Yan N: GLUT, SGLT, and SWEET: Structural and mechanistic investigations of the glucose transporters. Protein Sci 25: 546-558, 2016

44. Smolková K, Bellance N, Scandurra F, Génot E, Gnaiger E, Plecitá-Hlavatá L, Jezek P and Rossignol R: Mitochondrial bioenergetic adaptations of breast cancer cells to aglycemia and hypoxia. J Bioenerg Biomembr 42: 55-67, 2010.

45. Baffy G: Mitochondrial uncoupling in cancer cells: Liabilities and opportunities. Biochim Biophys Acta Bioenerg 1858: 655-664, 2017.

46. Liu Y, Cao Y, Zhang W, Bergmeier S, Qian Y, Akbar H, Colvin R, Ding J, Tong L, Wu S, et al: A small-molecule inhibitor of glucose transporter 1 downregulates glycolysis, induces cell-cycle arrest, and inhibits cancer cell growth in vitro and in vivo. Mol Cancer Ther 11: 1672-1682, 2012.

47. Vander Heiden MG, Cantley LC and Thompson CB: Understanding the Warburg effect: The metabolic requirements of cell proliferation. Science 324: 1029-1033, 2009.

48. Zambrano A, Molt M, Uribe E and Salas M: Glut 1 in cancer cells and the inhibitory action of resveratrol as a potential therapeutic strategy. Int J Mol Sci 20: 3374, 2019.

49. Samudio I, Harmancey R, Fiegl M, Kantarjian H, Konopleva M, Korchin B, Kaluarachchi K, Bornmann W, Duvvuri S, Taegtmeyer $\mathrm{H}$ and Andreeff M: Pharmacologic inhibition of fatty acid oxidation sensitizes human leukemia cells to apoptosis induction. J Clin Invest 120: 142-156, 2010.

50. Vélez J, Hail N Jr, Konopleva M, Zeng Z, Kojima K, Samudio I and Andreeff M: Mitochondrial uncoupling and the reprograming of intermediary metabolism in leukemia cells. Front Oncol 3: 67, 2013.

This work is licensed under a Creative Commons Attribution-NonCommercial-NoDerivatives 4.0 International (CC BY-NC-ND 4.0) License. 